\title{
Association of Consensus Molecular Subtypes and Molecular Markers With Clinical Outcomes in Patients With Metastatic Colorectal Cancer: Biomarker Analyses From LUME-Colon 1
}

\author{
Heinz-Josef Lenz, ${ }^{1}$ Guillem Argiles, ${ }^{2}$ Takayuki Yoshino, ${ }^{3}$ Sabine Tejpar, ${ }^{4,5}$
} Fortunato Ciardiello, ${ }^{6}$ Jürgen Braunger, ${ }^{7}$ Alexey V. Salnikov, ${ }^{7}$ Ogsen Gabrielyan, ${ }^{7}$ Ramona Schmid, ${ }^{7}$ Josef Höfler, ${ }^{8}$ Thomas Kitzing, ${ }^{7}$ Eric Van Cutsem ${ }^{5}$

\section{Abstract}

LUME-Colon 1 (NCT02149108) was a randomized, controlled, phase III study of nintedanib in 765 patients with advanced colorectal cancer. Pre-specified biomarker analyses investigated the association of colorectal cancer consensus molecular subtypes and tumor genomic/circulating biomarkers with clinical outcomes. Unclassified/mixed consensus molecular subtype was potentially predictive for nintedanib response; somatic mutations were highly concordant between tumor tissue and cell-free DNA.

Introduction: LUME-Colon 1 (NCT02149108) was a global, placebo-controlled phase III study of nintedanib in advanced colorectal cancer (CRC). Pre-specified biomarker analyses investigated the association of CRC consensus molecular subtypes (CMS) and tumor genomic and circulating biomarkers with clinical outcomes. Materials and Methods: Archival tumor tissue, cell-free DNA (cfDNA), and plasma samples were collected for genomic, transcriptomic, and proteomic analyses to investigate potential associations between CRC CMS and other biomarkers with nintedanib response and clinical outcomes. Results: Of the 765 treated patients, 735, 245, and 192 patient samples were analyzed in the circulating protein, tumor tissue, and cfDNA datasets, respectively. Patients were classified as CMS1 (1.7\%), CMS2 (27.7\%), CMS3 (0.9\%), CMS4 (51.5\%), or unclassified (18.2\%). Unclassified/mixed CMS was associated with longer overall survival (OS) with nintedanib vs. CMS2 or CMS4 (interaction $P$-value $=.0086$ ); no association was observed for CMS4. Gene expression-based pathway analysis revealed an association between vascular endothelial growth factor-related signaling and OS for nintedanib $(P=$ .0498). The most frequently detected somatic mutations were APC (72.0\% [tumor tissue] vs. 56.8\% [cfDNA]), TP53 ( $47.1 \%$ vs. $34.9 \%)$, KRAS ( $40.8 \%$ vs. $28.6 \%$ ), and PIK3CA (16.6\% vs. $11.5 \%)$; concordance rates were $>80 \%$. Median OS differences were observed for APC and TP53 mutations vs. wild-type in cfDNA, indicating a potential prognostic value. Circulating ANG-2, CA-9, CEACAM1, collagen-IV, IGFBP-1, ICAM-1, IL-8, and uPAR were potentially prognostic for both OS and progression-free survival. Conclusion: We demonstrated the feasibility of large-scale biomarker analyses and CMS classification within a global clinical trial, and identified signals suggesting a potential for greater nintedanib treatment response in the unclassified/mixed CMS subgroup, despite these tumors showing heterogeneous patterns of CMS mixtures. Our results revealed a high degree of

\footnotetext{
${ }^{1}$ University of Southern California Norris Comprehensive Cancer Center, Los Angeles, CA

${ }^{2}$ Vall d'Hebron University Hospital and Institute of Oncology (VHIO), Universitat Autònoma de Barcelona, Barcelona, Spain

${ }^{3}$ National Cancer Center Hospital East, Chiba, Japan

${ }^{4}$ Digestive Oncology Unit and Centre for Human Genetics, UZ Leuven, Belgium

${ }^{5}$ University Hospitals Leuven and KULeuven, Leuven, Belgium

${ }^{6}$ Dipartimento di Internistica Clinica e Sperimentale, Università degli Studi della Campania Luigi Vanvitelli, Naples, Italy
}

\footnotetext{
Boehringer Ingelheim Pharma GmbH \& Co. KG, Biberach, Germany ${ }^{8}$ Staburo GmbH, Munich, Germany

Submitted: Jun 16, 2020; Revised: Aug 21, 2020; Accepted: Sep 7, 2020

Address for correspondence: Heinz-Josef Lenz, MD, FACP, USC Norris Comprehensive Cancer Center, 1441 Eastlake Ave, NOR 3456, Los Angeles, CA 90089-9173 E-mail contact: lenz@med.usc.edu
} 


\section{Biomarker Analysis: Nintedanib in LUME-Colon 1}

concordance in somatic mutations between tumor tissue and cfDNA. Associations with prognosis for cfDNA somatic mutations, as well as several protein-based biomarkers, may warrant further investigation in future trials.

Clinical Colorectal Cancer, Vol. ๘, No. - - -- (c) 2020 Published by Elsevier Inc.

Keywords: Circulating biomarkers, Genomic biomarkers, Nintedanib, Predictive marker, Prognostic marker

\section{Introduction}

\section{Biomarkers in Colorectal Cancer}

Colorectal cancer (CRC) is one of the most common types of cancer; it is the third most common in men and the second most common in women. ${ }^{1}$ There are multiple genes and pathways known to be involved in its initiation, progression, and metastasis, resulting in a high degree of genetic heterogeneity. ${ }^{2,3}$ Transcriptomic characterization of CRC offers the potential to overcome some of the therapeutic challenges of genetic heterogeneity by identifying activated genes or pathways (ie, personalized therapeutic targets). ${ }^{3,4}$

Potential targets implicated in the initiation and progression of CRC include the WNT, RAS-MAPK, phosphoinositide 3-kinase $(\mathrm{PI} 3 \mathrm{~K})$, transforming growth factor beta (TGF- $\beta$ ), $\mathrm{p} 53$, and DNA mismatch-repair pathways. ${ }^{2}$ Their key role in the development and proliferation of CRC identifies these pathways (and related mutations) as being of potential utility as clinical biomarkers of treatment response (predictive biomarkers) and/or patient outcomes (prognostic biomarkers).

Adenomatous polyposis coli (APC) protein is a component of the Wnt/ $\beta$-catenin signaling pathway, which negatively regulates cell growth. Inactivation of APC appears to be an early (perhaps initiating) event in $\sim 70 \%$ to $80 \%$ of colorectal adenomas and carcinomas. However, APC has thus far shown limited potential as a clinical biomarker. ${ }^{2,5}$

$R A S$ and $B R A F$ mutations are present in an estimated $40 \%$ and $5 \%$ to $10 \%$ of patients with CRC, respectively, and are of interest as potential predictors of response to epidermal growth factor receptor (EGFR) therapy. 2,5

Mutations in the tumor protein p53 (TP53) gene are observed in $50 \%$ to $75 \%$ of CRCs. The TP53 protein has a regulatory role in cell growth arrest, DNA repair and apoptosis, oxidative stress, DNA damage, and cell aberrant proliferation; thus maintaining cell cycle homeostasis. Dysfunctions in TP53 have a critical role in oncogenesis in a wide range of tumor types, including CRC. 2,3,6

EGFR signaling has also been shown to play a critical role in tumor survival (including CRC), resulting in the development and approval of anti-EGFR targeted therapies. Human epidermal growth factor receptor 2 (HER2) signaling represents one potential mechanism of acquired resistance to anti-EGFR therapies. HER2 amplification has been detected in approximately $5 \%$ of $K R A S$ wildtype cancers, and there is evidence to support HER2 expression as a predictive biomarker for anti-HER2 treatment response. ${ }^{2,3,6}$

A leading cause of mutations found in CRC is dysfunction in the mismatch repair system, resulting in the well-established microsatellite instability (MSI) phenotype. MSI is reported in approximately $15 \%$ of all CRCs. Hypermutated CRC is highly correlated with
MSI, and is frequently observed together with $B R A F$ V600E mutation. The evidence to support MSI status as a biomarker for potential treatment response remains equivocal at this time. However, recent reports describe significantly better clinical outcomes in patients with MSI-high CRC compared with non-MSI CRC, suggesting potential utility for MSI status and hypermutated phenotypes as predictive markers for response to immunomodulating therapy.

The role of dysregulated angiogenesis is well-established in CRC, and is underpinned by the approval of several anti-angiogenic drugs that target vascular endothelial growth factor (VEGF) ligands (eg, VEGF-A, VEGF-B, VEGF-C, VEGF-D, and placenta growth factor $[\mathrm{PlGF}]$ ), their receptors (VEGFR1-3, Tie2 receptor tyrosine kinase), and other related proliferative pathways (platelet-derived growth factor receptor [PDGFR] and fibroblast growth factor receptor [FGFR]). However, cross-talk between VEGF and other crucial pathways is complex, and many patients demonstrate preexisting or acquired resistance to anti-angiogenic agents. ${ }^{8,9}$ The lack of a definitive predictive biomarker for anti-angiogenic treatment highlights the need for further biomarker analyses in largescale clinical trials. ${ }^{8}$

\section{Consensus Molecular Subtype (CMS) Classification}

The CMS classification system is defined by the pathways implicated in CRC. ${ }^{10}$ It comprises 4 distinct subgroups defined by gene expression levels: (1) CMS1 (MSI immune), hypermutated, microsatellite unstable, and strong immune activation; (2) CMS2 (canonical), epithelial, marked WNT and MYC signaling activation; (3) CMS3 (metabolic), epithelial and evident metabolic dysregulation; and (4) CMS4 (mesenchymal), prominent TGF $\beta$ activation, stromal invasion, and angiogenesis.

CMS has been shown to be a robust approach to CRC subtyping, and is of substantial interest for its potential as a predictive biomarker. ${ }^{11-15}$ Nevertheless, the ability of CMS to predict treatment response remains unclear owing to conflicting results from phase III trials, for example, in the CALGB/SWOG 80405 trial and FIRE3 trials. ${ }^{12,13}$ The predictive and prognostic values of CMS were evaluated in a heterogeneous population of patients with advanced CRC $(\mathrm{n}=581)$ in the CALGB/SWOG 80405 trial. $^{12}$ CMS subgroup was found to be associated with survival outcomes $(P<.001$ for both overall survival $[\mathrm{OS}]$ and progression-free survival $[\mathrm{PFS}])$. Patients in the CMS1 cohort who received bevacizumab had significantly longer OS than those treated with cetuximab $(P<.001)$ and, conversely, patients in the CMS2 cohort treated with cetuximab had significantly longer OS than patients treated with bevacizumab $(P=.0046) .{ }^{12}$ In the FIRE3 trial in patients with metastatic CRC $(\mathrm{n}=592)$, CMS was also found to be 
a predictor of OS $(P<.001)$ and PFS $(P<.001) .{ }^{13}$ However, there was no difference in OS among patients who received bevacizumab compared with those who received cetuximab in either CMS1 $(P=$ $.28)$ or CMS2 $(P=.40) .{ }^{13}$ These findings suggest that further research is needed in additional clinical trial populations to determine the role of CMS classification as a biomarker in identifying patients with metastatic CRC who are most likely to benefit from specific targeted therapies.

\section{LUME-Colon 1 Biomarker Analyses}

LUME-Colon 1 (NCT02149108) was a large-scale, global, randomized, double-blind, placebo-controlled, phase III trial investigating the efficacy and safety of nintedanib (vs. placebo) plus best supportive care in patients with metastatic CRC refractory to standard therapies. Results from the primary analysis have been published elsewhere. ${ }^{16}$

Here, we present the results from the biomarker analyses from LUME-Colon 1. At the time of study design, a comprehensive exploratory biomarker analysis was planned to provide molecular profiling data, based on targeted next-generation sequencing (NGS) of tumor tissue and cell-free DNA (cfDNA) samples, including a concordance analysis of mutations between tumor tissue and cfDNA samples. The analysis also included transcriptomic characterization of tumor tissue and an evaluation of protein biomarkers in plasma samples.

The approach taken with this global, phase III study provides an opportunity to explore whether a differential treatment effect was evident in any biomarker-defined subpopulations treated with nintedanib. The objective was to molecularly characterize the patients' tumors (ie, identify the frequency of potential clinical biomarkers) as well as to assess the utility of the CRC CMS classification system (and of other potential biomarkers proposed within the CRC literature) for their association with treatment response and/or clinical outcomes. The CMS4 subgroup was of particular interest as it is described as being associated with angiogenic pathway activation, and might, therefore, be more strongly associated with response to nintedanib (an anti-angiogenic agent) vs. other CMS subgroups.

\section{Materials and Methods}

\section{Study Design and Biomarker Sample Collection}

LUME-Colon 1 was a global, randomized, phase III study investigating the efficacy and safety of nintedanib (200 mg twice daily) vs. placebo in patients with advanced CRC. Detailed information about the trial design has been previously described. ${ }^{16}$

Plasma samples for circulating protein biomarker analysis were collected at randomization (before intake of study drug at cycle 1, visit 1), before intake of study drug at the start of treatment course 2 , and at the end of the trial.

Archival formalin-fixed, paraffin-embedded (FFPE) tumor tissue for genomic and transcriptomic profiling was collected and analyzed from all patients with sufficient and evaluable tumor tissue.

The final biomarker analysis in tumor tissue and cfDNA samples was performed on a subset of samples that were selected according to the following quality-based criteria: sample availability; tumor content $>40 \%$ (before macrodissection); RNA mean concentration $>20 \mathrm{ng} / \mu \mathrm{L}$; RNA DV 200 (percentage of RNA fragments $>200 \mathrm{nt}$ in size) $>28 \%$; availability of baseline ethylene diamine tetraacetic acid blood sample as germline reference for mutation profiling; and availability of cycle 1 , visit 1 plasma cfDNA sample.

\section{Outcome Measures in Biomarker Cohorts}

To assess the similarity of the biomarker cohorts and identify any sources of potential selection bias, the co-primary endpoints (OS and PFS) from the LUME-Colon 1 trial were evaluated for each biomarker cohort, and compared between cohorts and against the results for the overall trial population. Outcomes were evaluated as hazard ratios (HRs) with $95 \%$ confidence intervals (CIs) for both PFS and OS.

\section{Evaluation of Prognostic and Predictive Utility}

All investigational biomarkers were investigated for their association with patient outcomes (prognostic potential) and response to treatment (predictive potential).

Prognostic Analysis. To eliminate any potential confounding by treatment effect, evaluation of the prognostic potential of investigational biomarkers was conducted only in the placebo arm of the study. The analysis evaluated whether the presence or elevation of a particular biomarker at baseline, or classification within a specific CMS category, was associated with reduced or prolonged survival outcomes (OS and PFS).

Predictive Analysis. The predictive evaluation of investigational biomarkers included the nintedanib and placebo treatment arms, and assessed whether allocation to a particular biomarker subgroup (or to a specific CMS category) resulted in a relative treatment benefit in terms of OS and PFS in favor of nintedanib compared with placebo.

\section{Genomic Analysis}

Evaluation of the frequency of somatic cancer-associated mutations and hypermutations of DNA repair genes was performed in DNA samples isolated from FFPE tumor tissue, and from cfDNA isolated from plasma. The NGS panel was a self-designed Roche SeqCap EZ Choice panel comprising a total of 44 genes, including nintedanib target genes, commonly altered CRC genes, and somatic cancer-associated mutations (eg, APC, PIK3CA, and TP53), as well as hypermutations of DNA repair genes (eg, $M L H 1, M S H 2$, MSH3, MSH6, and POLE). See Supplemental Table 1 (in the online version at https://doi.org/10.1016/j.clcc.2020.09.003) for a complete list. Targeted DNAseq libraries were prepared from DNA isolated from FFPE tumor tissue as well as from cfDNA isolated from plasma samples using the SeqCap EZ together with the KAPA HyperPlus kit; sequencing was performed on an Illumina NextSeq 500 instrument using the NextSeq 500/550 Mid-Output v2 kit according to the manufacturer's instructions. Concordance between mutations detected in matching cfDNA and tumor tissue samples was also evaluated.

The tumor tissue dataset was used to further profile the LUMEColon 1 population by the CRC CMS classification based on transcriptome (RNAseq) data. RNAseq libraries were generated from RNA isolated from FFPE tumor tissue using the Illumina 


\section{Biomarker Analysis: Nintedanib in LUME-Colon 1}

TruSeq Stranded Total RNA LT (with Ribo-Zero Gold) kit and sequenced on an Illumina HiSeq 3000/4000 instrument using the HiSeq 3000/4000 SBS kit according to the manufacturer's instructions. Associations between the CRC CMS with PFS and OS were investigated, considering in particular the predictive potential of the angiogenesis-related CMS4 for response to nintedanib treatment.

\section{Plasma Protein Analysis}

Baseline plasma samples were used to identify potential plasmaderived protein biomarkers. In total, 55 soluble angiogenic factors were evaluated and analyzed using the Human AngiogenesisMAP (Myriad RBM), including: angiopoietin-2 (ANG-2), carbonic anhydrase 9 (CA-9), tenascin-C (TN-C), basic fibroblast growth factor (bFGF), VEGF A and D, VEGFR1-3, and PlGF. See Supplemental Table 2 (in the online version at https://doi.org/10. 1016/j.clcc.2020.09.003) for a complete list.

\section{Statistical Analysis}

Descriptive statistics were used to summarize data, including CMS distribution and mutational and pathway activation status. Kaplan-Meier methods were used in time-to-event analyses, and Cox regression was used to assess the predictive and prognostic potential of biomarkers. All analyses were exploratory; no additional confirmatory statistical analyses were conducted.

The association between plasma protein markers or molecular characteristics and response to nintedanib treatment was evaluated using the co-primary endpoints from the LUME-Colon 1 trial (centrally-assessed PFS and OS), and a treatment-by-biomarker interaction test based on 2 independent tests, both with a 2 -sided significance level of .05 . The prognostic potential of plasma protein and molecular characteristics was assessed in the placebo group via pairwise comparisons of individuals with/without the characteristic of interest. Subgroup analyses were only performed if $\geq 10$ patients were available per subgroup and per treatment.

Mutation profiling in cfDNA samples in the placebo-treated group were significantly associated with treatment benefit at a level of $P<.1$ (false discovery rate $[\mathrm{FDR}]$-adjusted $P$-value). Protein biomarkers were significantly associated with lack of treatment benefit at a level of $P<.01$ (FDR-adjusted $P$-value).

\section{CMS Analysis}

CMS classification analysis was conducted using the $\mathrm{R}$ package CMSclassifier, in line with published methodology (ie, centroidbased and default settings). ${ }^{10}$ Any dataset-specific refinements to the published methodology were agreed in consultation with the Swiss Institute of Bioinformatics, Lausanne, Switzerland. NGS gene expression data (expressed in transcripts per million reads) were $\log 2(x+1)$ transformed for use with the CMS classifier. Direct mapping of Ensemble IDs to Entrez IDs was not possible in approximately 10 cases.

\section{Results}

\section{Data Availability}

In total, 765 patients were treated in LUME-Colon 1 ( $\mathrm{n}=384$ nintedanib; $\mathrm{n}=381$ placebo). ${ }^{16}$ In this biomarker analysis, 735 patients contributed to the plasma protein dataset $(\mathrm{n}=368$
[95.8\%] nintedanib; $\mathrm{n}=367$ [96.3\%] placebo); 245 patients contributed to the tumor tissue dataset $(\mathrm{n}=125$ [32.6\%] nintedanib; $\mathrm{n}=120$ [31.5\%] placebo); and 192 patients contributed to the $\mathrm{cfDNA}$ dataset $(\mathrm{n}=104$ [27.1\%] nintedanib; $\mathrm{n}=88$ [23.1\%] placebo) (Figure 1). Matched tumor tissue and cfDNA data were available for concordance analysis for 118 patients $(n=57$ [14.8\%] nintedanib; $\mathrm{n}=61[16.0 \%]$ placebo).

\section{Baseline Characteristics}

There were no notable differences in patient demographics or clinical characteristics in the overall LUME-Colon 1 population (Table 1) or between different biomarker cohorts (see Supplemental Table 3 in the online version at https://doi.org/10.1016/j.clcc.2020. 09.003). There were no clinically meaningful differences in outcomes (PFS or OS) between any of the biomarker cohorts, or between any of the cohorts and the overall LUME-Colon 1 trial population (see Supplemental Table 4 in the online version at https://doi.org/10.1016/j.clcc.2020.09.003).

\section{CMS Categorization}

Of the 245 patient samples in the tumor tissue dataset, 231 $(94.3 \%)$ had evaluable RNA-Seq gene expression data and met the necessary quality criteria for CMS classification. Four (1.7\%) patients were CMS1; 64 (27.7\%) were CMS2; 2 (0.9\%) were CMS3, and 119 (51.5\%) were CMS4. The remaining 42 (18.2\%) patients were unclassifiable or of mixed phenotype by CMS categorization (hereafter referred to as 'unclassified') (see Supplemental Table 5 in the online version at https://doi.org/10.1016/j.clcc.2020.09.003).

CMS distribution was similar in the nintedanib and placebo groups. Demographic characteristics and oncological history were similar for the CMS subgroups and the overall tumor tissue cohort, with the exception of prior surgical tumor removal, which was more common in CMS4 patients than other groups (nintedanib: $95.0 \%$ vs. $82.5 \%$, respectively; placebo: $91.5 \%$ vs. $76.4 \%$, respectively).

Across all CMS subgroups, right-sided tumors were less common than left-sided tumors, with no right-sided tumors detected in the CMS1 and CMS3 subgroups and an incidence of 12.5\%, 9.2\%, and $9.5 \%$ for right-sided tumors in the CMS2, CMS4, and unclassified/mixed phenotype subgroups, respectively. The frequency of common CRC mutations varied across CMS subgroups, but APC mutations were consistently the most common, with an incidence ranging from $62.7 \%$ (47/75 samples) in CMS4 to $100 \%$ (3/3 and $1 / 1$ samples, respectively) in CMS1/CMS3. Only 1 class III $B R A F$ mutation was detected (in a patient with CMS2 CRC).

Owing to the low number of patients categorized as CMS1 or CMS3, the predictive/prognostic biomarker analyses omitted these groups and only considered the CMS2, CMS4, and unclassified subgroups.

The predictive biomarker analysis revealed an association between the unclassified CMS category and longer OS (interaction $P$ value $=.0086$ ); patients in this subgroup derived greater benefit from nintedanib treatment than those in either CMS2 or CMS4. The median OS in the unclassified/mixed subgroup was 11.1 months in the nintedanib arm vs. 4.7 months in the placebo arm (Figure 2). In contrast, the CMS4 subgroup (characterized by angiogenic pathway activation, among other features) was not associated with nintedanib treatment effect vs. non-CMS4 


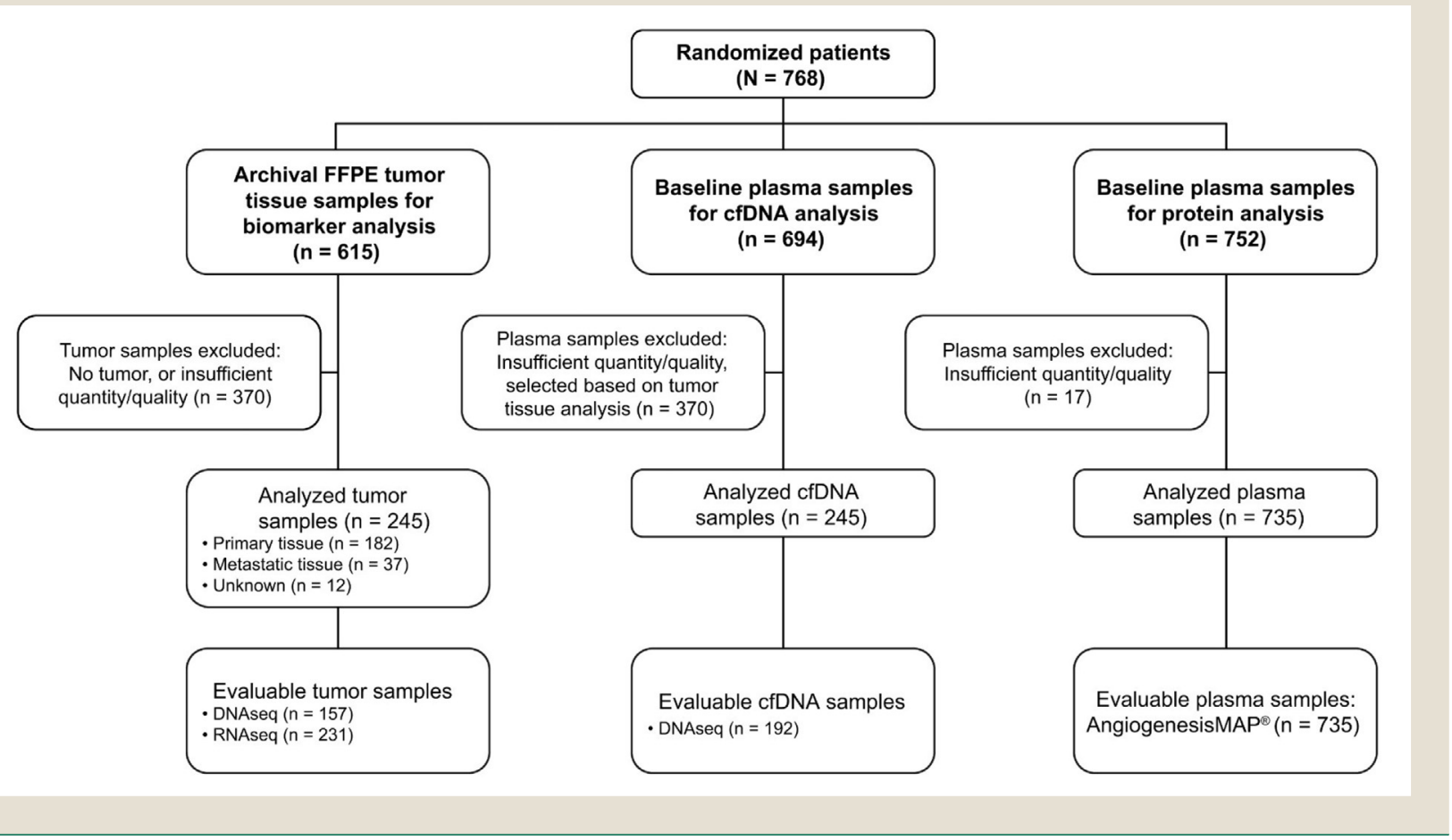

Abbreviations: cfDNA $=$ Cell-free DNA; FFPE $=$ formalin-fixed, paraffin-embedded

subgroups, in terms of either OS or PFS (interaction $P$-values .6634 and .8278, respectively) (see Supplemental Table 6 in the online version at https://doi.org/10.1016/j.clcc.2020.09.003).

\section{Signaling Pathways}

Gene expression data were used to evaluate pre-defined signaling pathway activation. The VEGF-related signaling pathway showed a predictive potential for nintedanib (for OS only; unadjusted $P$ value $=.0498$ ); based on an analysis of the placebo group, there was no prognostic potential for either OS or PFS. No prognostic/predictive associations were found for other signaling pathways, including activated vs. non-activated angiogenic pathways.

\section{Mutation Profiling}

Somatic mutations were assessed in 157 tumor tissue samples ( $\mathrm{n}=74$ nintedanib; $\mathrm{n}=83$ placebo) and $192 \mathrm{cfDNA}$ samples $(\mathrm{n}=$ 104 nintedanib; $\mathrm{n}=88$ placebo). The most frequently observed somatic mutations were in: $A P C(\mathrm{n}=113$ [72.0\%] tumor tissue vs. $\mathrm{n}=109$ [56.8\%] cfDNA); TP53 ( $\mathrm{n}=74$ [47.1\%] vs. $\mathrm{n}=67$ [34.9\%]); KRAS ( $\mathrm{n}=64[40.8 \%]$ vs. $\mathrm{n}=55$ [28.6\%]), and PIK3CA ( $\mathrm{n}=26[16.6 \%]$ vs. $\mathrm{n}=22[11.5 \%]$ ) (Figure 3). Detection rates for these genes were similar in both the nintedanib and placebo treatment groups. Of note, the frequency of $B R A F$ mutations in the analyzed patient cohort was very low; only 1 (0.6\%) BRAF D594G mutation was observed, which was detected in the tumor tissue as well as the plasma cfDNA samples of the patient.

Concordance was assessed in 118 patients with matched tumor and cfDNA samples, with a significant association $\left(R^{2}=0.9912\right.$;
$P<.0001)$ between the tumor tissue cohort and the cfDNA cohort for the 20 most commonly mutated genes (Figure 4). For the most frequently mutated genes (APC, TP53, KRAS, and PIK3CA), concordance was $82.2 \%, 81.4 \%, 87.3 \%$, and $89.8 \%$, respectively.

Differences in median OS were observed in cfDNA samples of patients with APC and TP53 mutations compared with patients with no mutations (in the placebo-treated group), at a significance level of $P<.1$ (FDR-adjusted $P$-value); suggestive of a potential prognostic value (Figure 5). The median OS for $A P C$ was 5.7 vs. 7.8 months (mutant vs. wild-type; HR, 1.74; 95\% CI, 1.05-2.89; $P=$ .0656). The median OS for TP53 was 5.7 vs. 7.3 months (HR, 1.93; 95\% CI, 1.14-3.25; $P=.0543$ ). The survival difference was not significant for PIK3CA: the median OS was 5.9 vs. 6.2 months (HR, 1.75; 95\% CI, 0.79-3.86; $P=.1683$ ). For KRAS, an association was observed for both OS and PFS at a significance level of $P<.1$; however, the median OS and PFS values were similar for mutated and wild-type patients: the median OS was 6.2 vs. 6.1 months (HR, 1.75; 95\% CI, 1.00-3.07; $P=.0658$ ), and the median PFS was 1.3 vs. 1.4 months (HR, 1.97; 95\% CI, 1.15-3.38; $P=.0546)$. No potentially prognostic signals were detected in the tumor tissue (FFPE) samples.

Hypermutation status (defined by the presence of any alteration in any of the DNA repair genes investigated) was not associated with either OS or PFS (see Supplemental Figure 1 in the online version at https://doi.org/10.1016/j.clcc.2020.09.003).

\section{Protein Biomarkers}

A panel of 55 circulating protein biomarkers was analyzed, which included regulators of growth, survival, angiogenesis, adhesion, and 


\section{Biomarker Analysis: Nintedanib in LUME-Colon 1}

Table 1 Baseline Characteristics in LUME-Colon 1 (Full Population and Biomarker Sub-population)

\begin{tabular}{|c|c|c|c|c|c|c|}
\hline & \multicolumn{3}{|c|}{ LUME-Colon 1, n (\%) } & \multicolumn{3}{|c|}{$\begin{array}{l}\text { LUME-Colon } 1 \text { Biomarker } \\
\text { Sub-population, }{ }^{a} \text { n (\%) }\end{array}$} \\
\hline & Placebo & Nintedanib & Total & Placebo & Nintedanib & Total \\
\hline No. patients & $382(100)$ & $386(100)$ & $768(100)$ & $120(100)$ & $125(100)$ & $245(100)$ \\
\hline \multicolumn{7}{|l|}{ Age, y } \\
\hline Mean & 61.1 & 61.0 & 61.1 & 59.9 & 59.8 & 59.9 \\
\hline Range & $23-83$ & $22-85$ & $22-85$ & $28-82$ & $30-85$ & $28-85$ \\
\hline \multicolumn{7}{|l|}{ Gender } \\
\hline Female & 164 (42.9) & $150(38.9)$ & 314 (40.9) & 59 (49.2) & $51(40.8)$ & $110(44.9)$ \\
\hline Male & 218 (57.1) & $236(61.1)$ & 454 (59.1) & $61(50.8)$ & 74 (59.2) & $135(55.1)$ \\
\hline \multicolumn{7}{|l|}{ Geographical region } \\
\hline Asia & $98(25.7)$ & 95 (24.6) & $193(25.1)$ & $32(26.7)$ & $31(24.8)$ & $63(25.7)$ \\
\hline Australia & $12(3.1)$ & $14(3.6)$ & $26(3.4)$ & $6(5.0)$ & $6(4.8)$ & $12(4.9)$ \\
\hline North America & $29(7.6)$ & $34(8.8)$ & $63(8.2)$ & $6(5.0)$ & $8(6.4)$ & $14(5.7)$ \\
\hline Western Europe & $186(48.7)$ & $183(47.4)$ & $369(48.0)$ & $54(45.0)$ & $60(48.0)$ & $114(46.5)$ \\
\hline Rest of world & 57 (14.9) & $60(15.5)$ & 117 (15.2) & $22(18.3)$ & $20(16.0)$ & $42(17.1)$ \\
\hline \multicolumn{7}{|l|}{ Race } \\
\hline Asian & $104(27.2)$ & $97(25.1)$ & 201 (26.2) & 34 (28.3) & $31(24.8)$ & $65(26.5)$ \\
\hline White & $268(70.2)$ & $279(72.3)$ & 547 (71.2) & $82(68.3)$ & $91(72.8)$ & $173(70.6)$ \\
\hline Other & $3(0.8)$ & $5(1.3)$ & $8(1.0)$ & $0(0.0)$ & $1(0.8)$ & $1(0.4)$ \\
\hline Missing $^{b}$ & $9(2.4)$ & $5(1.3)$ & $14(1.8)$ & $4(3.3)$ & $2(1.6)$ & $6(2.4)$ \\
\hline \multicolumn{7}{|l|}{ ECOG PS at baseline } \\
\hline 0 & $142(37.2)$ & $162(42.0)$ & $304(39.6)$ & $42(35.0)$ & $48(38.4)$ & $90(36.7)$ \\
\hline 1 & $240(62.8)$ & $223(57.8)$ & $463(60.3)$ & $78(65.0)$ & $76(60.8)$ & $154(62.9)$ \\
\hline$\geq 2$ & $0(0.0)$ & $1(0.3)$ & $1(0.1)$ & $0(0.0)$ & $1(0.8)$ & $1(0.4)$ \\
\hline \multicolumn{7}{|l|}{ Primary tumor location ${ }^{C}$} \\
\hline Left & $295(77.2)$ & $298(77.2)$ & $593(77.2)$ & $91(75.8)$ & $95(76.0)$ & $186(75.9)$ \\
\hline Right & $86(22.5)$ & $88(22.8)$ & $174(22.7)$ & $29(24.2)$ & $30(24.0)$ & $59(24.1)$ \\
\hline Missing & $1(0.3)$ & $0(0.0)$ & $1(0.1)$ & $0(0.0)$ & $0(0.0)$ & $0(0.0)$ \\
\hline \multicolumn{7}{|l|}{ Time since first histologic diagnosis, mos } \\
\hline Mean (SD) & $47.4(29.9)$ & $46.5(29.5)$ & $46.9(29.7)$ & $43.2(27.4)$ & $43.1(27.8)$ & $43.1(27.6)$ \\
\hline Range & $2-155$ & $6-245$ & $2-245$ & $8-138$ & $6-208$ & $6-208$ \\
\hline \multicolumn{7}{|c|}{ Number of lines of previous anticancer therapies } \\
\hline 1 & $11(2.9)$ & $7(1.8)$ & $18(2.3)$ & $4(3.3)$ & $4(3.2)$ & $8(3.3)$ \\
\hline 2 & 75 (19.6) & $82(21.2)$ & $157(20.4)$ & $23(19.2)$ & $30(24.0)$ & $53(21.6)$ \\
\hline 3 & 109 (28.5) & $90(23.3)$ & $199(25.9)$ & 39 (32.5) & $26(20.8)$ & $65(26.5)$ \\
\hline 4 & $70(18.3)$ & $87(22.5)$ & $157(20.4)$ & $19(15.8)$ & $33(26.4)$ & $52(21.2)$ \\
\hline 5 & $54(14.1)$ & $59(15.3)$ & $113(14.7)$ & $10(8.3)$ & $19(15.2)$ & $29(11.8)$ \\
\hline$\geq 6$ & $63(16.5)$ & $61(15.8)$ & $124(16.1)$ & $25(20.8)$ & $13(10.4)$ & $38(15.5)$ \\
\hline \multicolumn{7}{|l|}{ Location of metastatic sites at screening } \\
\hline Lung & $305(79.8)$ & $316(81.9)$ & $621(80.9)$ & $95(79.2)$ & $106(84.8)$ & $201(82.0)$ \\
\hline Liver & $266(69.6)$ & $277(71.8)$ & $543(70.7)$ & $79(65.8)$ & $86(68.8)$ & $165(67.3)$ \\
\hline Lymph nodes & $191(50.0)$ & $184(47.7)$ & $375(48.8)$ & $63(52.5)$ & $54(43.2)$ & $117(47.8)$ \\
\hline Peritoneum/omentum/malignant ascites & $84(22.0)$ & $93(24.1)$ & $177(23.0)$ & $23(19.2)$ & $28(22.4)$ & $51(20.8)$ \\
\hline Bone & $46(12.0)$ & $35(9.1)$ & $81(10.5)$ & $16(13.3)$ & $6(4.8)$ & $22(9.0)$ \\
\hline Other & $120(31.4)$ & $143(37.0)$ & $263(34.2)$ & 37 (30.8) & $38(30.4)$ & $75(30.6)$ \\
\hline
\end{tabular}

Abbreviations: ECOG PS = Eastern Cooperative Oncology Group performance status; FFPE = formalin-fixed, paraffin-embedded; SD = standard deviation.

abased on patient subset with archival FFPE tumor tissue.

Includes data from countries where collection of patient race data is not permitted.

${ }^{c}$ Right-sided defined as cecum, ascending colon, and transverse colon. Left-sided defined as descending colon, sigmoid colon, and rectum. 


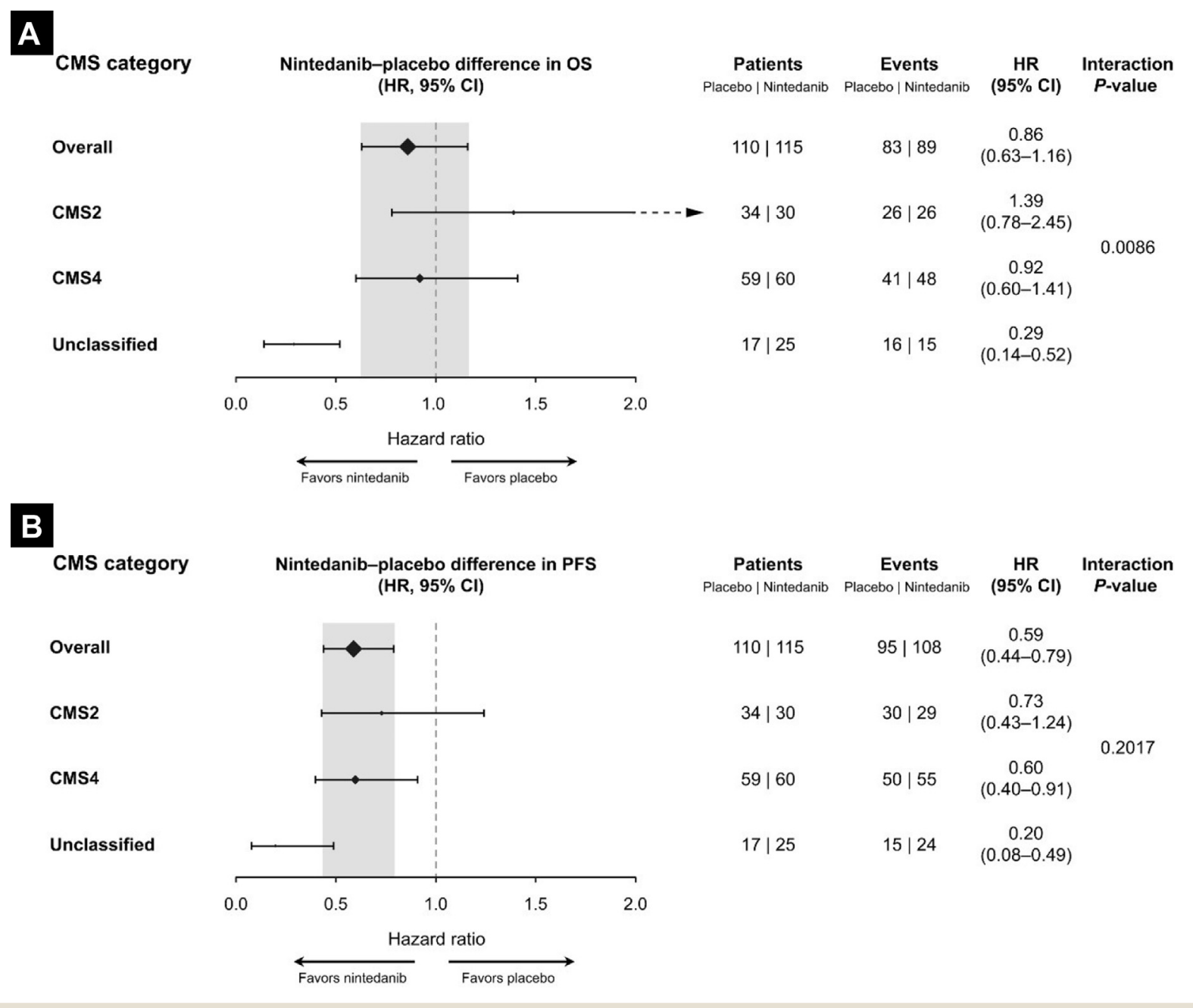

Only subgroups comprising $\geq 10$ patients were included in the analysis.

Abbreviations: $\mathrm{Cl}=$ confidence interval; $\mathrm{CMS}=$ consensus molecular subtype; $\mathrm{HR}=$ hazard ratio; $\mathrm{OS}=$ overall survival; PFS = progression-free survival.

other oncogenic pathways; most of which were present at detectable levels. At a significance level of $P<.01$ (FDR-adjusted $P$-value), 8 of the 55 protein biomarkers were found to be prognostic for both OS and PFS: ANG-2; CA-9; carcinoembryonic antigen-related cell adhesion molecule 1 (CEACAM1); collagen IV; insulin-like growth factor-binding protein (IGFBP)-1; intercellular adhesion molecule (ICAM)-1, IL-8, and urokinase receptor (uPAR). For these 8 proteins, high plasma concentrations at baseline were correlated with shorter OS and PFS. In addition to those 8 proteins, 21 protein biomarkers were prognostic for OS only. No protein biomarkers were predictive for PFS or OS at a significance level $<.01$ (FDRadjusted interaction $P$-value).

\section{Discussion}

\section{Distribution of CMS Subgroups in LUME-Colon 1}

There were notable differences between the CMS distribution in the LUME-Colon 1 biomarker population and that reported in the literature (Figure 6): CMS1 (1.7\% observed vs. 9\%-17\% reported); CMS2 (27.7\% vs. $37 \%-50 \%)$; CMS3 (0.9\% vs. $2 \%-13 \%)$; CMS4 $(51.5 \%$ vs. $23 \%-45 \%)$; and unclassified/mixed (18.2\% vs. $0 \%-$ $13 \%)$. Importantly, the observed proportion of unclassified/mixed CMS patients is higher in our study than previously reported; although recent data from the ColoType classifier report approximately 16\% unclassified samples, and data from PETACC-3 show $>20 \%$ unclassified samples. ${ }^{17}$ As the CMS classification was conducted in accordance with published methods, ${ }^{10}$ it is important to consider other potential factors for these differences, which might include the range of tumor sample sites that contributed to the analysis, the range of disease stages, and the number of previous lines of treatment within the analyzed population at baseline.

The strict patient eligibility criteria of LUME-Colon 1 (advanced, refractory CRC) compared with those of the CMS consensus paper (which analyzed a broad range of patients with CRC across 18 datasets) might have resulted in a different distribution of patient 


\section{Biomarker Analysis: Nintedanib in LUME-Colon 1}

Figure 3 Frequency of Somatic Mutations in LUME-Colon 1 (FFPE vs. cfDNA Samples) and Other Publically Available Datasets

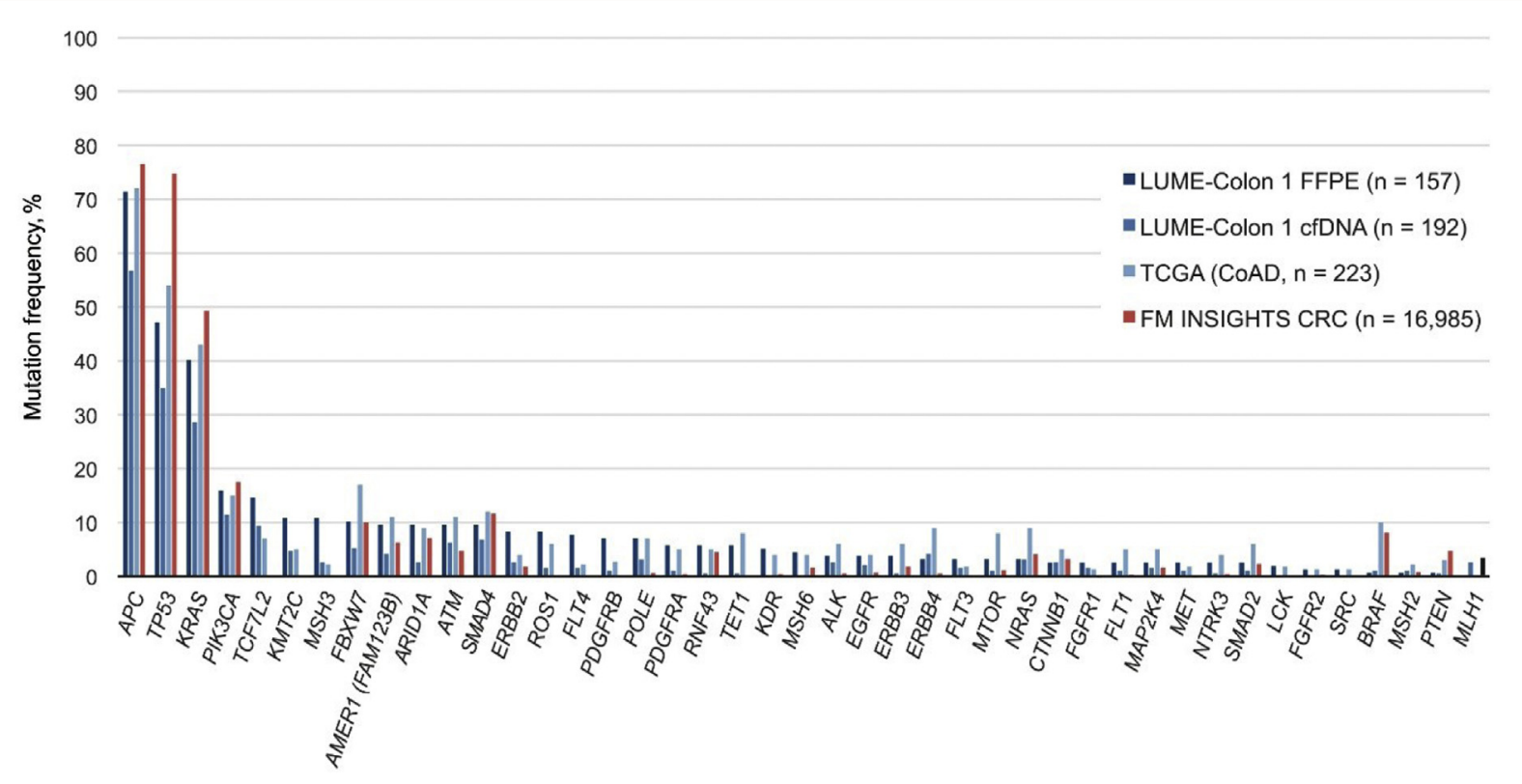

Abbreviations: cfDNA $=$ cell-free DNA; CRC $=$ colorectal cancer; FFPE $=$ formalin-fixed, paraffin-embedded; TCGA $=$ The Cancer Genome Atlas Network.

characteristics. ${ }^{10,16}$ Within the tumor tissue cohort, the majority of samples were taken from primary tumors $(\mathrm{n}=182 ; 79 \%)$, but a proportion were also taken from metastases $(\mathrm{n}=37 ; 16 \%)$ or from an unknown source $(\mathrm{n}=12 ; 5 \%)$. All patients had stage IV disease; approximately one-half of the patients in LUME-Colon 1 were randomized more than 36 months after their first histologic diagnosis. At the time of randomization, $25 \%$ of the patients had received 1 to 2 previous lines of treatment; $48 \%$ of the patients had received 3 to 4 previous lines of treatment; and $27 \%$ of patients had received $\geq 5$ previous lines of treatment. Both factors - chemo-refractory setting and analysis of metastatic tissue - have been reported to impact on the distribution of CMS as well as the number of unclassified/mixed samples. ${ }^{18,19}$ Finally, an unusually low frequency of $B R A F$ mutations was observed in this trial $(0.6 \%$ observed vs. $5 \%-10 \%$ typically reported). ${ }^{2}$ No instances of $B R A F \mathrm{~V} 600$ mutations were observed in the LUME-Colon 1 population.

In turn, this altered distribution of CMS subgroups could have affected the biomarker analysis and limited its accuracy. It is also important to note that, although the biomarker analysis was performed on archival FFPE tumor tissue samples, the observed distribution of the CMS from the LUME-Colon 1 analysis may reflect the prognostic effect of the individual subtypes, and thus be more similar to the chemo-refractory setting and analysis of metastases. Indeed, this may also explain the observed low frequency of CMS1 (and BRAF mutation frequency) in the dataset, as the prognostic effect of $B R A F$ mutation may have affected enrollment into the study.

\section{Predictive Potential of CMS Subgroups}

The observed association between the unclassified/mixed CMS subgroup and longer OS highlights a gap in our knowledge of CRC, though should be interpreted with caution owing to the low number of patients ( $\mathrm{n}=42$ unclassified/mixed patients). The literature describes as many as $13 \%$ of patients being of unclassified or mixed CMS category. ${ }^{10}$ In this study, the proportion of samples in the unclassified/mixed subgroup may have been higher owing to the factors described above. Forcing the samples into 1 of the 4 CMS groups by applying 'nearest CMS' classification resulted in distribution of the 'unclassified' samples across CMS1 $(n=6)$, CMS2 $(n=12)$, CMS3 $(n=3)$, and CMS4 $(n=21)$; there was no association for any of the adjusted CMS groups with clinical parameters (PFS or OS).

Somewhat surprisingly, there was no association between CMS4 (which is characterized by angiogenic pathway activation) and either OS or PFS, as compared with other CMS subgroups. However, a separate analysis of VEGF pathway activation, based on gene expression profile data, did suggest that nintedanib treatment was associated with OS.

\section{Mutational Analyses in Tumor Tissue and cfDNA}

Results from the mutational analysis are consistent with those reported in the literature. Mutations in KRAS were detected in $40.8 \%$ (tumor tissue) and $28.6 \%$ (cfDNA) of patients with CRC in LUME-Colon 1 vs. an estimated $40 \%$ of patients with CRC in published studies. ${ }^{2,5}$ Meanwhile, TP53 mutations were observed in $47.1 \%$ (tumor tissue) and $34.9 \%$ (cfDNA) of patients with CRC, as compared with $50 \%$ to $75 \%$ of patients with CRC in published studies. $^{2,3,6}$

The LUME-Colon 1 biomarker analysis revealed a high concordance of mutations detected in tumor tissue and cfDNA samples. This supports the use of plasma samples in routine clinical practice as a means to classify CRC by mutation status to monitor response to treatment in real-time, and detect both minimal residual 
Figure 4 Correlation Between Missense Mutation Frequencies in Tumor Tissue and cfDNA Samples

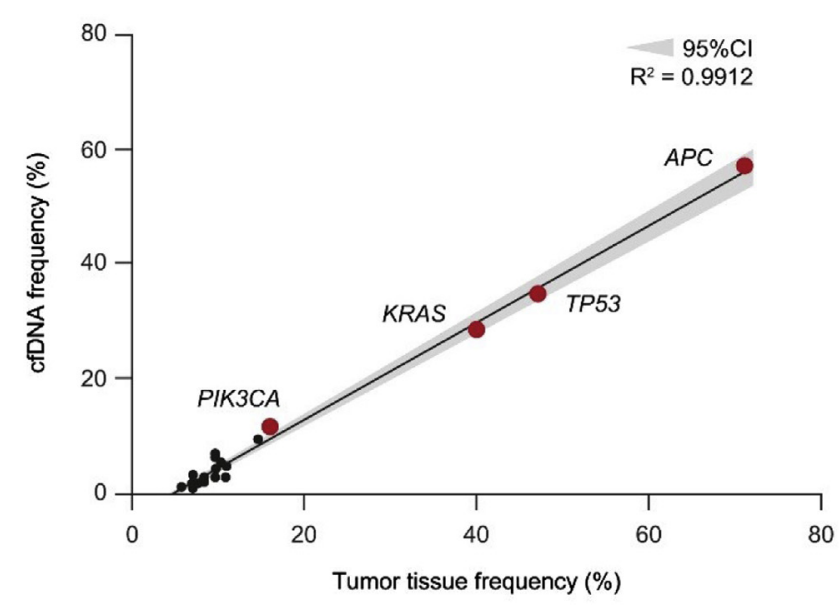

\begin{tabular}{l|c} 
Gene & $\begin{array}{c}\text { Concordance of mutation frequency } \\
\text { between cfDNA and tumor tissue }\end{array}$ \\
\hline APC & $82.2 \%$ \\
\hline$T P 53$ & $81.4 \%$ \\
\hline KRAS & $87.3 \%$ \\
\hline PIK3CA & $89.8 \%$
\end{tabular}

Graph shows missense mutation frequencies in tumor tissue and cfDNA for the 20 most frequently mutated genes observed in tumor tissue samples. Abbreviations: cfDNA = cell-free DNA; $\mathrm{Cl}=$ confidence interval.

disease and the emergence of targeted treatment-resistant clones. ${ }^{20}$ As the routine collection of plasma isolated cfDNA during serial assessments becomes more established in clinical practice, patients with CRC undergoing genomic profiling may increasingly benefit from this minimally invasive procedure. ${ }^{20}$ Nevertheless, concordance between cfDNA and tumor samples fell short of $100 \%$,

\section{Figure 5 Prognostic Potential of Selected Mutations Measured in cfDNA on OS (Placebo Group). TP53 (A), APC (B), KRAS, (C) and PIK3CA (D)}

A

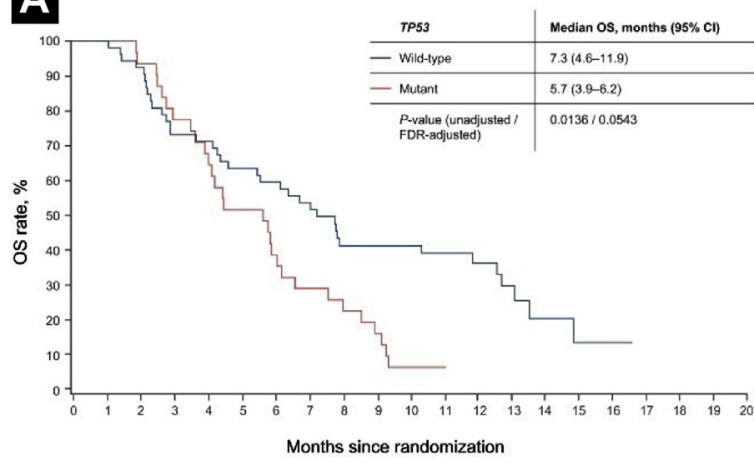

C

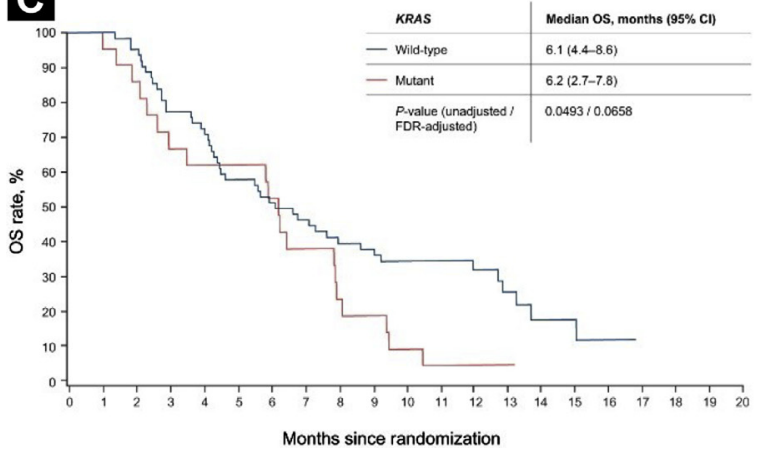

B

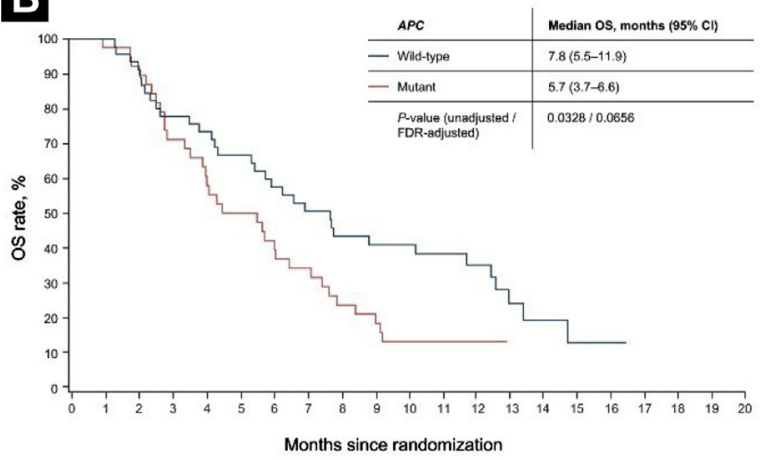

D

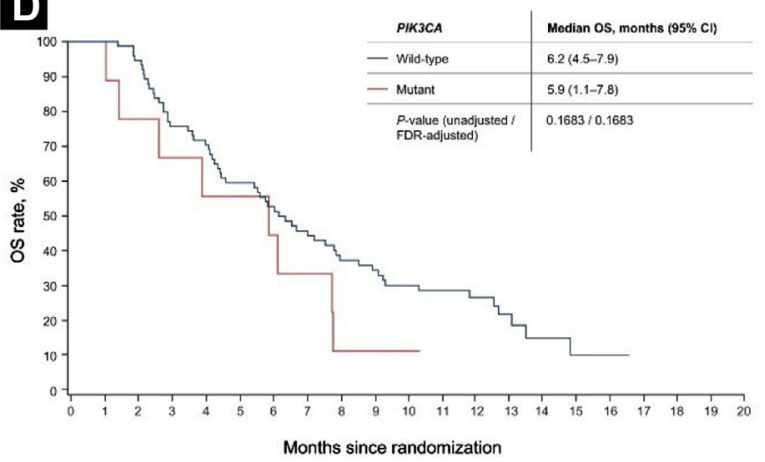

Abbreviations: $\mathrm{Cl}=$ confidence interval; $\mathrm{FDR}=$ false discovery rate; $\mathrm{OS}=$ overall survival. 


\section{Biomarker Analysis: Nintedanib in LUME-Colon 1}

\section{Figure 6 CMS Distribution in LUME-Colon 1 and Other Published CRC Clinical Trials}

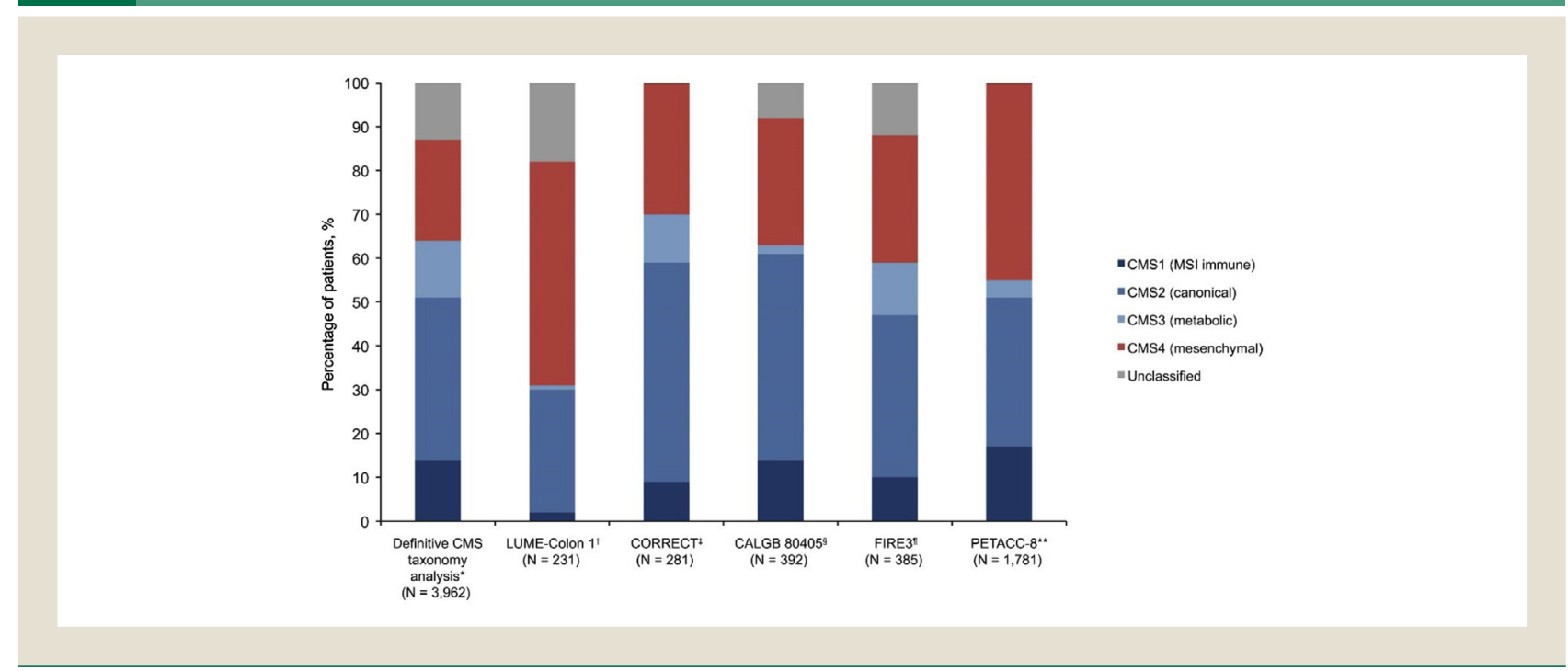

Adapted from results in published references. ${ }^{10-14}$

Abbreviations: CMS = consensus molecular subtype; FOLFIRI = folinic acid, flurouracil, and irinotecan chemotherapy; FOLFOX = folinic acid, flurouracil, and oxaliplatin chemotherapy; MSI = microsatellite instability.

*Stages I-IV; '`Stage IV; nintedanib vs. placebo; CMS analysis by RNAseq; ${ }^{\ddagger}$ Stage IV; regorafenib vs. placebo; CMS analysis by Affymetrix; ${ }^{\circledR}$ Stage IV; bevacizumab + chemotherapy vs. cetuximab + chemotherapy; CMS analysis by nanoString (custom designed); "Stage IV; bevacizumab + FOLFIRI vs. cetuximab + FOLFIRl; CMS analysis by Affymetrix (ALMAC Xcel); **Stage III; FOLFOX vs. FOLFOX + cetuximab; CMS analysis by nanoString (196 genes).

which is reflected in the observation that only APC and TP53 were identified as having potential prognostic value via the cfDNA cohort analysis, and none of the most frequently mutated genes (APC, PIK3CA, KRAS, or TP53) were found to have either predictive or prognostic utility in the tumor tissue cohort analysis. KRAS mutations in cfDNA were found to be potentially prognostic for both PFS and OS, although the median PFS and OS values were similar for mutated and non-mutated patients. A recent publication reports a similar concordance rate of $85 \%$ between blood and tumor tissue, as well as a prognostic effect of KRAS mutation for OS, which is strongest in patients with concordant KRAS mutation status in both blood and tumor tissue samples. ${ }^{21}$

These results should be interpreted with caution, owing to the limited number of samples involved in our analysis; it should also be noted that patients with higher levels of cfDNA typically have a greater tumor burden and worse outcomes. ${ }^{22,23}$ Differences such as this may be the result of incomplete overlap between the cfDNA and tumor tissue cohorts (only $\sim 61 \%$ of patients from the tumor tissue set were included in the cfDNA sample set) and also the fact that, although plasma samples were collected from patients on entry to the LUME-Colon 1 trial, the tumor tissue samples were archival and (in most cases) collected several years before start of the trial, giving rise to the potential for divergence.

\section{Analysis of Protein Biomarkers}

Several protein biomarkers were found to be associated with both OS and PFS (ANG-2, CA-9, CEACAM1, collagen IV, IGFBP-1, ICAM-1, IL-8, and UPAR), including members of angiogenesis, adhesion, and proliferation pathways. Other exploratory biomarker studies in patients with metastatic CRC have identified similar protein markers or signatures. ${ }^{8,24-26}$ For example: circulating angiogenic profiles (which signify tumor hypoxia) seem to be associated with worse outcomes ${ }^{25}$; in patients treated with bevacizumab, plasma cytokine and angiogenic factors are associated with PFS and $\mathrm{OS}^{24}$; in the CORRECT trial of regorafenib, a greater effect on OS was observed in patients with high TIE-1 expression levels (a factor that supports tumor-associated endothelial survival) than those with low TIE-1 levels ${ }^{26}$; and in a study of sunitinib and sorafenib, levels of angiogenic factors can change in response to treatment, perhaps reflecting a susceptibility of intratumoral blood vessels to anti-angiogenic treatment. ${ }^{25}$

Given the poor treatment outcomes associated with metastatic CRC and the complexity of angiogenesis pathways, as well as the dearth of validated response markers, more biomarker analyses from large-scale clinical trials will be essential to conclusively identify circulating protein biomarkers that can robustly predict for response to anti-angiogenic agents such as nintedanib. ${ }^{8}$

\section{Limitations}

This was an exploratory biomarker analysis and not powered to detect statistical significant or clinically significant associations between investigational biomarkers and clinical endpoints. The sample cohorts used in the mutational and genomic analyses involved a subset $(\mathrm{n}=245 ; 32 \%)$ of the total patients randomized in the LUME-Colon 1 trial $(\mathrm{N}=768)$, with sample selection limited by parameters, such as availability of good quality archival tumor tissue and paired pre-treatment plasma cfDNA samples, and other quality control measures. Nevertheless, the consistency of OS and PFS outcomes observed for the different biomarker cohorts, and for the overall LUME-Colon 1 population, provides confidence that biomarker sample selection 
did not result in any clinically meaningful selection bias. Finally, as LUME-Colon 1 did not meet its primary objective, it is challenging to definitively identify biomarkers associated with treatment response.

\section{Conclusion}

In conclusion, analysis of the CRC CMS using RNAseq was feasible in this large-scale, global, phase III clinical trial - although there were notable differences between the carefully defined LUMEColon 1 trial population and the more varied patients included in the original CRC CMS sub-group analysis. Of note, the predictive signal observed with the unclassified/mixed CMS group may warrant further research once future refinements of the disease classification are available; for example, with potential intra-CMS subgroups and with better characterization of samples with mixed phenotypes. Predictive and/or prognostic potential was observed for APC and TP53 mutations and for a range of protein biomarkers, suggesting avenues for future research in patients with advanced CRC. Finally, the high degree of concordance between tumor tissue and cfDNA in the mutational analysis strengthens the rationale for the use of liquid biopsies to help guide treatment decisions in the clinic.

\section{Clinical Practice Points}

- Several anti-angiogenic drugs are approved for the treatment of CRC. However, cross-talk between proliferative pathways is complex; many patients display resistance, revealing a need for biomarker analyses in large-scale clinical trials.

- LUME-Colon 1 (NCT02149108) was a placebo-controlled, phase III study of nintedanib in 765 patients with advanced CRC. Pre-planned biomarker analyses investigated the association of CRC CMS and tumor genomic and circulating biomarkers with clinical outcomes. Evaluation of CMS4 was of particular interest owing to its association with angiogenic pathway activation and nintedanib's anti-angiogenic mechanism of action. Overall, 735, 245, and 192 patient samples contributed to the plasma protein, tumor tissue, and cfDNA datasets, respectively.

- CMS distribution in the LUME-Colon 1 biomarker population differed from the published literature: CMS1 (1.7\%); CMS2 (27.7\%); CMS3 (0.9\%); CMS4 (51.5\%); and unclassified/ mixed (18.2\%). An association between unclassified CMS and OS in the nintedanib-treated group highlights an important gap in our knowledge of CRC. Although there was no association with CMS4, VEGF pathway activation was identified as a marker for nintedanib treatment effect. Mutations in $A P C$, TP53, KRAS, and PIK3CA (in cfDNA) were associated with OS. Circulating ANG-2, CA-9, CEACAM1, collagen-IV, IGFBP-1, ICAM-1, IL-8, and UPAR were potentially prognostic for both OS and PFS.

- In summary, we conducted a large-scale biomarker analysis and CMS classification within a global clinical trial setting. Unclassified/mixed CMS was potentially predictive for nintedanib response; somatic mutations were highly concordant between tumor tissue and cfDNA; several biomarkers may warrant further investigation in future trials.

\section{Acknowledgments}

This work was conducted in collaboration with Professor Pratyaksha Wirapati (Swiss Institute of Bioinformatics, Lausanne, Switzerland) who provided methodological expertise on the CMS classification.

Boehringer Ingelheim provided financial support for the LUMEColon 1 study, including study analysis and manuscript development. Editorial support was provided by Alison Chisholm and Nirmal Jethwa (Syneos Health; London, UK).

\section{Disclosure}

H.J. Lenz has received personal fees, grants, and non-financial support from Bayer; personal fees and grants from Merck KG and Boehringer Ingelheim; and personal fees from Roche. T. Yoshino's institution has received research funds from Novartis Pharma KK, MSD KK, Sumitomo Dainippon Pharma Co, Ltd, Chugai Pharmaceutical Co, Ltd, Sanofi KK, Daiichi Sankyo Co, Ltd, Parexel International Inc, Ono Pharmaceutical Co, Ltd, and GlaxoSmithKline KK. S. Tejpar has received personal fees, institutional grants, and non-financial support from Bayer and Merck KGaA, and personal and institutional fees from Boehringer Ingelheim, Roche, Amgen, MSD, and Servier. F. Ciardiello's institution has received financial interests, financial support for clinical trials, or contracted research from Merck Serono, Roche, Symphogen, Array; direct research funding as the principal investigator for institutional research projects from Amgen, Bayer, Merck Serono, Roche, and Ipsen; and honoraria or consultation fees for speaker, consultancy or advisory roles from Amgen, Bayer, Bristol-Myers Squibb, Celgene, Merck Serono, Pfizer, Roche, and Servier. J. Höfler has received personal fees from Boehringer Ingelheim Pharma GmbH \& Co. KG during the conduct of the study for biostatistical analyses. J. Braunger, A. Salnikov, O. Gabrielyan, R. Schmid, and T. Kitzing are employees of Boehringer Ingelheim. E. Van Cutsem's institution has received research funds from Amgen, Bayer, Boehringer Ingelheim, Celgene, Ipsen, Lilly, Merck, Merck KGaA, Novartis, Roche, Sanofi, and Servier, and has had an advisory role for Bayer, Lilly, Roche, and Servier. G. Argiles declares no potential conflicts of interest.

\section{Supplemental Data}

Supplemental figure and tables accompanying this article can be found in the online version at https://doi.org/10.1016/j.clcc.2020. 09.003 .

\section{References}

1. International Agency for Research on Cancer. Cancer today: data visualization tools for exploring the global cancer burden in 2018. Available at: http://gco.iarc. fr/today/home. Accessed October 30, 2019.

2. Fearon ER. Molecular genetics of colorectal cancer. Annu Rev Pathol 2011; 6:479-507.

3. Zarkavelis G, Boussios S, Papadaki A, Katsanos KH, Christodoulou DK, Pentheroudakis G. Current and future biomarkers in colorectal cancer. Ann Gastroenterol 2017; 30:613-21.

4. Dienstmann R, Vermeulen L, Guinney J, Kopetz S, Tejpar S, Tabernero J. Consensus molecular subtypes and the evolution of precision medicine in colorectal cancer. Nat Rev Cancer 2017; 17:79-92. 


\section{Biomarker Analysis: Nintedanib in LUME-Colon 1}

5. George B, Kopetz S. Predictive and prognostic markers in colorectal cancer. Cur Oncol Rep 2011; 13:206-15.

6. Boussios S, Ozturk MA, Moschetta M, et al. The developing story of predictive biomarkers in colorectal cancer. J Pers Med 2019; 9:12.

7. Yuza K, Nagahashi M, Watanabe S, Takabe K, Wakai T. Hypermutation and microsatellite instability in gastrointestinal cancers. Oncotarget 2017; 8:112103-15.

8. Mousa L, Salem ME, Mikhail S. Biomarkers of angiogenesis in colorectal cancer. Biomark Cancer 2015; 7(Suppl 1):13-9.

9. Battaglin F, Puccini A, Intini R, et al. The role of tumor angiogenesis as a therapeutic target in colorectal cancer. Expert Rev Anticancer Ther 2018; 18:251-66.

10. Guinney J, Dienstmann R, Wang X, et al. The consensus molecular subtypes of colorectal cancer. Nat Med 2015; 21:1350-6.

11. Teufel M, Schwenke S, Seidel H, et al. Molecular subtypes and outcomes in regorafenib-treated patients with metastatic colorectal cancer (mCRC) enrolled in the CORRECT trial. J Clin Oncol 2015; 33(15 Suppl):3558.

12. Lenz H-J, Ou F-S, Venook AP, et al. Impact of consensus molecular subtype on survival in patients with metastatic colorectal cancer: results from CALGB/SWOG 80405 (alliance). J Clin Oncol 2019; 37:1876-85.

13. Stintzing S, Wirapati $\mathrm{P}$, Lenz $\mathrm{H}-\mathrm{J}$, et al. Consensus molecular subgroups (CMS) of colorectal cancer (CRC) and first-line efficacy of FOLFIRI plus cetuximab or bevacizumab in the FIRE3 (AIO KRK-0306) trial. J Clin Oncol 2017; 35(15 Suppl):3510.

14. Marisa L, Ayadi M, Balogoun R, et al. Clinical utility of colon cancer molecula subtypes: validation of two main colorectal molecular classifications on the PETACC-8 phase III trial cohort. J Clin Oncol 2017; 35 (15 Suppl):3509.

15. Okita A, Takahashi S, Ouchi K, et al. Consensus molecular subtypes classification of colorectal cancer as a predictive factor for chemotherapeutic efficacy against metastatic colorectal cancer. Oncotarget 2018; 9:18698-711.

16. Van Cutsem E, Yoshino T, Lenz HJ, et al. Nintedanib for the treatment of patients with refractory metastatic colorectal cancer (LUME-Colon 1): a phase III, international, randomized, placebo-controlled study. Ann Oncol 2018; 29:1955-63.
17. Buechler SA, Stephens MT, Hummon AB, et al. ColoType: a forty gene signature for consensus molecular subtyping of colorectal cancer tumors using wholegenome assay or targeted RNA-sequencing. Sci Rep 2020; 10:12123.

18. Alderdice M, Richman SD, Gollins S, et al. Prospective patient stratification into robust cancer-cell intrinsic subtypes from colorectal cancer biopsies. J Pathol 2018 245:19-28.

19. Fontana E, Eason K, Cervantes A, Salazar R, Sadanandam A. Context matters: consensus molecular subtypes of colorectal cancer as biomarkers for clinical trials. Ann Oncol 2019; 30:520-7.

20. Osumi H, Shinozaki E, Yamaguchi K, Zembutsu H. Clinical utility of circulating tumor DNA for colorectal cancer. Cancer Sci 2019; 110:1148-55.

21. Mardinian K, Okamura R, Kato S, Kurzrock R. Temporal and spatial effects and survival outcomes associated with concordance between tissue and blood KRAS alterations in the pan-cancer setting. Int J Cancer 2020; 146: 566-76.

22. Gold B, Cankovic M, Furtado LV, Meier F, Gocke CD. Do circulating tumor cells, exosomes, and circulating tumor nucleic acids have clinical utility? J Mol Diagn 2015; 17:209-24.

23. Schwarzenbach H, Hoon DSB, Pantel K. Cell-free nucleic acids as biomarkers in cancer patients. Nat Rev Cancer 2011; 11:426-37.

24. Bai L, Wang F, Zhang D, et al. A plasma cytokine and angiogenic factor (CAF) analysis for selection of bevacizumab therapy in patients with metastatic colorectal cancer. Sci Rep 2015; 5:17717.

25. Xu W, Puligandla M, Manola J, et al. Angiogenic factor and cytokine analysis among patients treated with adjuvant VEGFR TKIs in resected renal cell carcinoma. Clin Cancer Res 2019; 25:6098-106.

26. Tabernero J, Lenz H-J, Siena S, et al. Analysis of circulating DNA and protein biomarkers to predict the clinical activity of regorafenib and assess prognosis in patients with metastatic colorectal cancer: a retrospective, exploratory analysis of the CORRECT trial. Lancet Oncol 2015; 16:937-48. 


\section{Supplemental Data}

\section{Supplemental Figure 1 Mutation Profile (A) and prognostic Analysis (B) of OS and PFS in Putative Hypermutated ${ }^{\S}$ vs. Non- hypermutated Tumors}

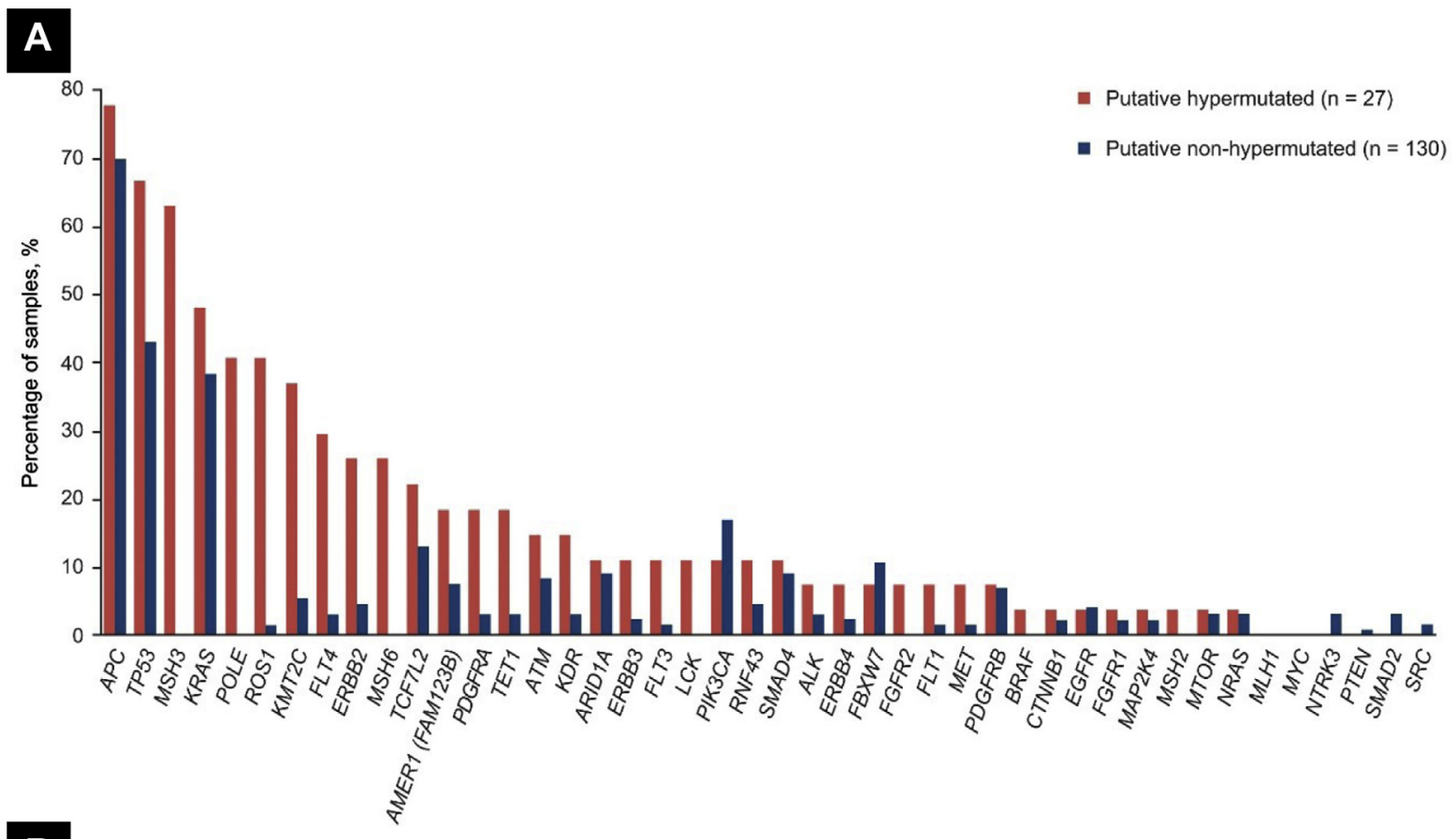

B

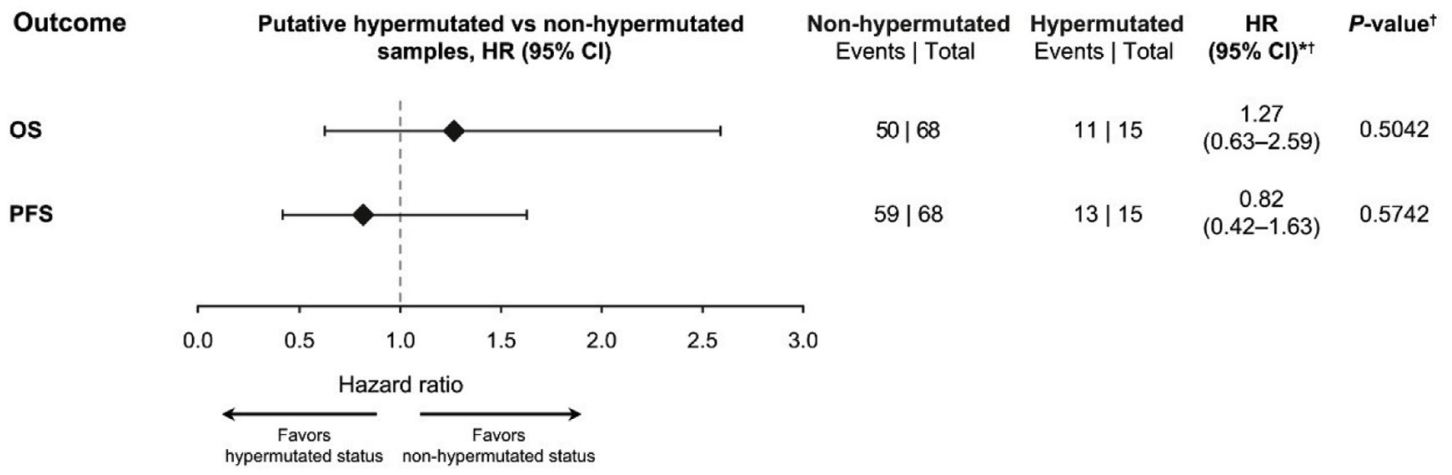

*HR $<1$ favored hypermutated samples. ${ }^{\dagger} \mathrm{HR}, \mathrm{Cl}$, and $P$-value were obtained from a model adjusted for treatment, regorafenib pre-treatment, time from onset of metastatic disease until randomization, and region. ${ }^{\S}$ Hypermutation status was defined by the presence of a mutation in at least one of the DNA repair genes included in the gene panel (ie, MLH1, MSH2, MSH3, MSH6, or POLE). Abbreviations: $\mathrm{Cl}=$ confidence interval; $\mathrm{HR}=$ hazard ratio; $\mathrm{OS}=$ overall survival; $\mathrm{PFS}=$ progression-free survival. 


\section{Biomarker Analysis: Nintedanib in LUME-Colon 1}

\section{Supplemental Table 1 Genes Included in the LUME-Colon 1 Targeted NGS Panel}

\begin{tabular}{|c|c|c|c|}
\hline ALK & FBXW7 & MET & PIKЗCA \\
\hline AMER1 (FAM123B) & FGFR1 $^{a}$ & MLH1 & POLE \\
\hline$A P C$ & FGFR2 $^{2}$ & MSH2 & PTEN \\
\hline$A R I D 1 A$ & $F L T 1^{a}$ & MSH3 & RNF43 \\
\hline ATM & $\mathrm{FLT3}^{\mathrm{a}}$ & MSH6 & ROS1 \\
\hline BRAF & $F L T 4^{a}$ & MTOR & SMAD2 \\
\hline CTNNB1 & $K D R^{a}$ & MYC & SMAD4 \\
\hline EGFR & KMT2C & NRAS & $S R C^{a}$ \\
\hline ERBB2 & KRAS & NTRK3 & TCF7L2 \\
\hline ERBB3 & $\operatorname{LCK}^{a}$ & PDGFRA $^{a}$ & TET1 \\
\hline ERBB4 & MAP2K4 & PDGFRB $^{a}$ & TP53 \\
\hline
\end{tabular}

In total, 44 genes were evaluated, consisting of nintedanib target genes, commonly altered CRC genes, and somatic cancer-associated mutations. This was a custom-designed gene panel covering all exons of each individual gene and utilizing a Roche SeqCap EZ hybridization capture-based target enrichment method. Sequencing was performed on DNA samples extracted from archival FFPE tumor tissue, plasma-isolated cfDNA, and gDNA isolated from whole blood, which permitted the identification of somatic alterations and enabled comparison between tumor tissue and cfDNA. Abbreviations: cfDNA = cell-free DNA; CRC = colorectal cancer; gDNA = genomic DNA; FFPE = formalin-fixed, paraffin-embedded; NGS = next-generation sequencing.

${ }^{a}$ Nintedanib target genes. 
Supplemental Table 2 Proteins Included in the Plasma Biomarker Analysis

\section{Ckine}

Analyte Name

Angiopoietin-1

Angiopoietin-2

Cancer antigen 15-3

Carbonic anhydrase 9

Carcinoembryonic antigen-related cell adhesion molecule 1

Collagen IV

Decorin

Endoglin

Epidermal growth factor

Epidermal growth factor receptor

Fatty acid-binding protein, adipocyte

Fibroblast growth factor, basic

Granulocyte-macrophage colony-stimulating factor

Heparin-binding EGF-like growth factor

Hepatocyte growth factor receptor

Hepsin

Human epidermal growth factor receptor 2

Insulin-like growth factor-binding protein 1

Insulin-like growth factor-binding protein 2

Intercellular adhesion molecule 1

Interferon gamma

Interferon-inducible T-cell alpha chemoattractant

Interleukin-1 alpha

Interleukin-1 beta

Interleukin-10

Interleukin-2

Interleukin-6

Interleukin-8

Kallikrein 5

Kallikrein-7

Macrophage inflammatory protein-3 beta

Macrophage inflammatory protein-1 alpha

Macrophage inflammatory protein-1 beta

Macrophage migration inhibitory factor

Maspin

Matrix metalloproteinase-3

Matrix metalloproteinase- 9

Mesothelin

Monocyte chemotactic protein 1

Placenta growth factor

Platelet endothelial cell adhesion molecule

Platelet-derived growth factor BB

Stem cell factor

Stromal cell-derived factor-1

Tenascin-C

Transforming growth factor alpha

Tumor necrosis factor alpha

Tumor necrosis factor beta

Urokinase-type plasminogen activator receptor

Vascular endothelial growth factor

Vascular endothelial growth factor B

\begin{tabular}{|c|c|c|c|}
\hline $\begin{array}{l}\text { Abbreviated } \\
\text { Name }\end{array}$ & $\begin{array}{c}\text { UniProtKB/ } \\
\text { SwissProt ID }\end{array}$ & HGNC & Type \\
\hline & 000585 & CCL21 & Cytokine \\
\hline ANG-1 & Q15389 & ANGPT1 & Growth factor \\
\hline ANG-2 & 015123 & ANGPT2 & Growth factor \\
\hline CA-15-3 & NA & NA & NA \\
\hline CA-9 & Q16790 & CA9 & Enzyme \\
\hline \multirow[t]{4}{*}{ CEACAM1 } & P13688 & CEACAM1 & Other \\
\hline & P02462/Q01955 & COL4A1/A3 & Other \\
\hline & P07585 & DCN & Other \\
\hline & P17813 & ENG & TM receptor \\
\hline EGF & P01133 & EGF & Growth factor \\
\hline EGFR & P00533 & EGFR & Kinase \\
\hline FABP, adipocyte & P15090 & FABP4 & Transporter \\
\hline bFGF & P09038 & FGF2 & Growth factor \\
\hline GM-CSF & P04141 & CSF2 & Cytokine \\
\hline HB-EGF & Q99075 & HBEGF & Growth factor \\
\hline \multirow[t]{2}{*}{ HGF receptor } & P08581 & MET & Kinase \\
\hline & P05981 & HPN & Peptidase \\
\hline HER-2 & P04626 & ERBB2 & Linase \\
\hline IGFBP-1 & P08833 & IGFBP1 & Other \\
\hline IGFBP-2 & P18065 & IGFBP2 & Other \\
\hline ICAM-1 & P05362 & ICAM1 & TM receptor \\
\hline IFN-gamma & P01579 & IFNG & Cytokine \\
\hline ITAC & 014625 & CXCL11 & Cytokine \\
\hline IL-1 alpha & P01583 & $\| \mathrm{L} 1 \mathrm{~A}$ & Cytokine \\
\hline IL-1 beta & P01584 & IL1B & Cytokine \\
\hline IL-10 & P22301 & IL10 & Cytokine \\
\hline IL-2 & P60568 & IL2 & Cytokine \\
\hline IL-6 & P05231 & IL6 & Cytokine \\
\hline IL-8 & P10145 & CXCL8 & Cytokine \\
\hline KLK5 & Q9Y337 & KLK5 & Peptidase \\
\hline KLK7 & P49862 & KLK7 & Peptidase \\
\hline MIP-3 beta & Q99731 & CCL19 & Cytokine \\
\hline MIP-1 alpha & P10147 & CCL3 & Cytokine \\
\hline MIP-1 beta & P13236 & CCL4 & Cytokine \\
\hline \multirow[t]{2}{*}{ MIF } & P14174 & MIF & Cytokine \\
\hline & P36952 & SERPINB5 & Other \\
\hline MMP-3 & P08254 & MMP3 & Peptidase \\
\hline MMP-9 & P14780 & MMP9 & Peptidase \\
\hline MSLN & Q13421 & MSLN & Other \\
\hline MCP-1 & P13500 & CCL2 & Cytokine \\
\hline PIGF & P49763 & PGF & Growth factor \\
\hline PECAM-1 & P16284 & PECAM1 & Other \\
\hline PDGF-BB & P01127 & PDGFB & Growth factor \\
\hline SCF & P21583 & KITLG & Growth factor \\
\hline SDF-1 & P48061 & CXCL12 & Cytokine \\
\hline TN-C & P24821 & TNC & Other \\
\hline TGF-alpha & P01135 & TGFA & Growth factor \\
\hline TNF-alpha & P01375 & TNF & Cytokine \\
\hline TNF-beta & P01374 & LTA & Cytokine \\
\hline UPAR & Q03405 & PLAUR & TM receptor \\
\hline VEGF & P15692 & VEGFA & Growth factor \\
\hline VEGF-B & P49765 & VEGFB & Growth factor \\
\hline
\end{tabular}


Biomarker Analysis: Nintedanib in LUME-Colon 1

\section{Supplemental Table 2 Continued}

\begin{tabular}{l|c|c|c|c} 
Analyte Name & $\begin{array}{c}\text { Abbreviated } \\
\text { Name }\end{array}$ & $\begin{array}{c}\text { UniProtKB/ } \\
\text { SwissProt ID }\end{array}$ & HGNC & Type \\
Vascular endothelial growth factor D & VEGF-D & 043915 & FIGF & Growth factor \\
\hline Vascular endothelial growth factor receptor 1 & VEGFR-1 & P17948 & FLT1 & Kinase \\
\hline Vascular endothelial growth factor receptor 2 & VEGFR-2 & P35968 & KDR & Kinase \\
\hline Vascular endothelial growth factor receptor 3 & VEGFR-3 & P35916 & FLT4 & TM receptor \\
\hline
\end{tabular}

Abbreviations: $\mathrm{NA}=$ no annotation provided by Myriad RBM; TM = transmembrane. 
Supplemental Table 3 Baseline Characteristics (Overall Population, and Plasma Protein, Tumor Tissue, cfDNA, and Tumor Tissue/cfDNA Concordance Sub-Populations)

\begin{tabular}{|c|c|c|c|c|c|c|c|c|c|c|}
\hline & \multicolumn{2}{|c|}{ Total Population, n (\%) } & \multicolumn{2}{|c|}{$\begin{array}{c}\text { Plasma Protein } \\
\text { Sub-population, } \mathbf{n}(\%)\end{array}$} & \multicolumn{2}{|c|}{$\begin{array}{c}\text { Tumor Tissue } \\
\text { Sub-population, } \mathbf{n}(\%)\end{array}$} & \multicolumn{2}{|c|}{ cfDNA Sub-population, n (\%) } & \multicolumn{2}{|c|}{ Concordance Population, ${ }^{a}$ n (\%) } \\
\hline & $\begin{array}{l}\text { Nintedanib } \\
(\mathrm{n}=386)\end{array}$ & $\begin{array}{l}\text { Placebo } \\
(\mathrm{n}=382)\end{array}$ & $\begin{array}{l}\text { Nintedanib } \\
(\mathrm{n}=368)\end{array}$ & $\begin{array}{l}\text { Placebo } \\
(n=367)\end{array}$ & $\begin{array}{l}\text { Nintedanib } \\
(n=125)\end{array}$ & $\begin{array}{l}\text { Placebo } \\
(n=120)\end{array}$ & $\begin{array}{l}\text { Nintedanib } \\
(\mathrm{n}=104)\end{array}$ & $\begin{array}{l}\text { Placebo } \\
(\mathrm{n}=88)\end{array}$ & $\begin{array}{l}\text { Nintedanib } \\
(\mathrm{n}=\mathbf{8 8})\end{array}$ & $\begin{array}{l}\text { Placebo } \\
(\mathrm{n}=82)\end{array}$ \\
\hline Mean age, y (range) & $61.0(22-85)$ & $61.1(23-83)$ & $61.0(28-85)$ & $61.4(28-82)$ & $59.8(30.0-85)$ & $59.9(28-82)$ & $60.8(28-85)$ & $60.5(28-82)$ & $60.9(34-85)$ & $61(28-82)$ \\
\hline Gender (male) & $236(61.1)$ & $218(57.1)$ & $221(60.1)$ & $211(57.5)$ & $74(59.2)$ & $61(50.8)$ & $59(56.7)$ & $47(53.4)$ & $49(55.7)$ & $43(52.4)$ \\
\hline \multicolumn{11}{|l|}{ Race } \\
\hline Caucasian & $279(72.3)$ & $268(70.2)$ & $261(70.9)$ & $253(68.9)$ & $91(72.8)$ & $82(68.3)$ & $84(80.8)$ & $62(70.5)$ & $69(78.4)$ & $57(69.5)$ \\
\hline Asian & $97(25.1)$ & $104(27.2)$ & $97(26.4)$ & $104(28.3)$ & $31(24.8)$ & $34(28.3)$ & $19(18.3)$ & $25(28.4)$ & $18(20.5)$ & $24(29.3)$ \\
\hline Other & $5(1.3)$ & $3(0.8)$ & $5(1.4)$ & $3(0.8)$ & $1(0.8)$ & $0(0.0)$ & $0(0.0)$ & $0(0.0)$ & $0(0.0)$ & $0(0.0)$ \\
\hline Missing $^{b}$ & $5(1.3)$ & $9(2.4)$ & $5(1.4)$ & $9(2.5)$ & $2(1.6)$ & $4(3.3)$ & $1(1.0)$ & $1(1.1)$ & $1(1.1)$ & $1(1.2)$ \\
\hline \multicolumn{11}{|l|}{ ECOG PS at baseline } \\
\hline 0 & $162(42.0)$ & $142(37.2)$ & $152(41.3)$ & $135(36.8)$ & $48(38.4)$ & $42(35.0)$ & $41(39.4)$ & $32(36.4)$ & 34 (38.6) & $30(36.6)$ \\
\hline 1 & $223(57.8)$ & $240(62.8)$ & $215(58.4)$ & $232(63.2)$ & $76(60.8)$ & $78(65.0)$ & $62(59.6)$ & 56 (63.6) & $53(60.2)$ & $52(63.4)$ \\
\hline$>1$ & $1(0.3)$ & $0(0.0)$ & $1(0.3)$ & $0(0.0)$ & $1(0.8)$ & $0(0.0)$ & $1(1.0)$ & $0(0.0)$ & $1(1.1)$ & $0(0.0)$ \\
\hline \multicolumn{11}{|c|}{ Time from onset of metastatic disease until randomization, mos } \\
\hline$<24$ & $108(28.0)$ & $110(28.8)$ & $102(27.7)$ & $109(29.7)$ & $45(36.0)$ & $36(30.0)$ & $34(32.7)$ & $29(33.0)$ & $32(36.4)$ & $27(32.9)$ \\
\hline$\geq 24$ & $278(72.0)$ & $272(71.2)$ & $266(72.3)$ & $258(70.3)$ & $80(64.0)$ & $84(70.0)$ & $70(67.3)$ & $59(67.0)$ & $56(63.6)$ & $55(67.1)$ \\
\hline \multicolumn{11}{|l|}{ Primary site of disease } \\
\hline Colon & $227(58.8)$ & $206(53.9)$ & $217(59.0)$ & $198(54.0)$ & $77(61.6)$ & $68(56.7)$ & $62(59.6)$ & $51(60.0)$ & $55(62.5)$ & $47(57.3)$ \\
\hline Rectum & $130(33.7)$ & $154(40.3)$ & $124(33.7)$ & $148(40.3)$ & 39 (31.2) & $46(38.3)$ & $33(31.7)$ & 31 (35.2) & 28 (31.8) & $30(36.6)$ \\
\hline Cecum & $29(7.5)$ & $21(5.5)$ & $27(7.3)$ & $20(5.4)$ & $9(7.2)$ & $6(5.0)$ & $9(8.7)$ & $6(6.8)$ & $5(5.7)$ & $5(6.1)$ \\
\hline Other/unknown & $0(0.0)$ & $1(0.3)$ & $0(0.0)$ & $1(0.3)$ & $0(0.0)$ & $0(0.0)$ & $0(0.0)$ & $0(0.0)$ & $0(0.0)$ & $0(0.0)$ \\
\hline \multicolumn{11}{|l|}{ Previous treatments } \\
\hline $\begin{array}{l}\text { Prior lines of systematic } \\
\text { anticancer therapies, } \\
\text { mean (SD) }\end{array}$ & $3.9(1.8)$ & $3.9(1.8)$ & $3.9(1.8)$ & $3.8(1.8)$ & 3.7 (1.79) & $3.8(1.76)$ & 3.8 (1.95) & $3.8(1.78)$ & $3.8(1.88)$ & $3.8(1.74)$ \\
\hline$\geq 3$ & $297(76.9)$ & $296(77.5)$ & $285(77.4)$ & $285(77.7)$ & $91(72.8)$ & 93 (77.5) & $77(74.0)$ & $66(75.0)$ & 65 (73.9) & $629(75.6)$ \\
\hline Oxaliplatin & 386 (100.0) & $382(100.0)$ & $368(100.0)$ & 367 (100.0) & $125(100.0)$ & $120(100.0)$ & $104(100.0)$ & $88(100.0)$ & $88(100.0)$ & $82(100.0)$ \\
\hline Fluoropyrimidine & $386(100.0)$ & $382(100.0)$ & $368(100.0)$ & 367 (100.0) & $125(100.0)$ & $120(100.0)$ & $104(100.0)$ & $88(100.0)$ & $88(100.0)$ & $82(100.0)$ \\
\hline Irinotecan & $386(100.0)$ & $382(100.0)$ & $368(100.0)$ & 367 (100.0) & $125(100.0)$ & $120(100.0)$ & $104(100.0)$ & $88(100.0)$ & $88(100.0)$ & $82(100.0)$ \\
\hline Bevacizumab & $368(95.3)$ & $370(96.9)$ & 352 (95.7) & 355 (96.7) & 119 (95.2) & 117 (97.5) & 99 (95.2) & $86(97.7)$ & 84 (95.5) & 81 (98.8) \\
\hline Aflibercept & $48(12.4)$ & 47 (12.3) & 45 (12.2) & $44(12.0)$ & $18(14.4)$ & 15 (12.5) & $12(11.5)$ & $10(11.4)$ & $10(11.4)$ & $8(9.8)$ \\
\hline Regorafenib & $141(36.5)$ & $144(37.7)$ & $135(36.7)$ & $140(38.1)$ & $41(32.8)$ & $41(34.2)$ & 39 (37.5) & $28(31.8)$ & $33(37.5)$ & $26(31.7)$ \\
\hline Trifluridine/tipiracil & 55 (14.2) & $53(13.9)$ & 55 (14.9) & $53(14.4)$ & $11(8.8)$ & $11(9.2)$ & $9(8.7)$ & $9(10.2)$ & $8(9.1)$ & $8(9.8)$ \\
\hline
\end{tabular}




\begin{tabular}{|c|c|c|c|c|c|c|c|c|c|c|}
\hline & \multicolumn{2}{|c|}{ Total Population, n (\%) } & \multicolumn{2}{|c|}{$\begin{array}{c}\text { Plasma Protein } \\
\text { Sub-population, } \mathbf{n}(\%)\end{array}$} & \multicolumn{2}{|c|}{$\begin{array}{c}\text { Tumor Tissue } \\
\text { Sub-population, } \mathbf{n}(\%)\end{array}$} & \multicolumn{2}{|c|}{ cfDNA Sub-population, n (\%) } & \multicolumn{2}{|c|}{ Concordance Population, ${ }^{\mathrm{a}} \mathrm{n}(\%)$} \\
\hline & $\begin{array}{l}\text { Nintedanib } \\
(\mathrm{n}=386)\end{array}$ & $\begin{array}{l}\text { Placebo } \\
(\mathrm{n}=382)\end{array}$ & $\begin{array}{l}\text { Nintedanib } \\
(\mathrm{n}=368)\end{array}$ & $\begin{array}{l}\text { Placebo } \\
(n=367)\end{array}$ & $\begin{array}{l}\text { Nintedanib } \\
(\mathrm{n}=125)\end{array}$ & $\begin{array}{l}\text { Placebo } \\
(n=120)\end{array}$ & $\begin{array}{l}\text { Nintedanib } \\
(n=104)\end{array}$ & $\begin{array}{l}\text { Placebo } \\
(n=88)\end{array}$ & $\begin{array}{l}\text { Nintedanib } \\
(\mathrm{n}=\mathbf{8 8})\end{array}$ & $\begin{array}{l}\text { Placebo } \\
\text { (n = 82) }\end{array}$ \\
\hline Other & $331(85.8)$ & $331(86.6)$ & $314(85.3)$ & $317(86.4)$ & $101(80.8)$ & $103(85.8)$ & $76(86.4)$ & $84(80.8)$ & $70(79.5)$ & $70(85.4)$ \\
\hline Prior radiotherapy & $104(26.9)$ & $132(34.6)$ & $97(26.4)$ & $123(33.5)$ & $31(24.8)$ & $44(36.7)$ & $27(26.0)$ & $28(31.8)$ & $22(25.0)$ & $27(32.9)$ \\
\hline $\begin{array}{l}\text { KRAS wild-type and } \\
\text { other RAS } \\
\text { wild-type patients only }\end{array}$ & $\begin{array}{l}\text { Nintedanib } \\
(\mathrm{n}=158)\end{array}$ & $\begin{array}{c}\text { Placebo } \\
(n=176)\end{array}$ & $\begin{array}{l}\text { Nintedanib } \\
(\mathrm{n}=150)\end{array}$ & $\begin{array}{c}\text { Placebo } \\
(\mathrm{n}=171)\end{array}$ & $\begin{array}{l}\text { Nintedanib } \\
(\mathrm{n}=40)\end{array}$ & $\begin{array}{c}\text { Placebo } \\
(\mathrm{n}=55)\end{array}$ & $\begin{array}{c}\text { Nintedanib } \\
(\mathrm{n}=39)\end{array}$ & $\begin{array}{c}\text { Placebo } \\
(\mathrm{n}=40)\end{array}$ & $\begin{array}{c}\text { Nintedanib } \\
(\mathrm{n}=32)\end{array}$ & $\begin{array}{c}\text { Placebo } \\
(\mathrm{n}=38)\end{array}$ \\
\hline $\begin{array}{l}\text { Prior cetuximab or } \\
\text { panitumumab }\end{array}$ & $158(100.0)$ & $175(99.4)$ & $150(100.0)$ & $170(99.4)$ & $40(100.0)$ & $55(100.0)$ & $39(100.0)$ & $40(100.0)$ & $32(100.0)$ & $38(100.0)$ \\
\hline
\end{tabular}

Abbreviations: cfDNA = cell-free DNA; ECOG PS = Eastern Cooperative Oncology Group performance status; NS = not specified; SD = standard deviation.

Abbreviations: cfDNA = cell-free DNA; ECOG PS = Eastern Coopr
aSubpopulation with matched tumor tissue and cfDNA samples.

asubpopulation with matched tumor tissue and crDNA samples.
${ }^{b}$ Can include patients living in a country where race is not allowed to be collected. 


\section{Supplemental Table 4 Co-primary Endpoints (Overall Population and Biomarker Subgroups)}

\begin{tabular}{|c|c|c|c|}
\hline Cohort & $\begin{array}{c}\text { Patients } \\
\text { Placebo I Nintedanib }\end{array}$ & $\begin{array}{c}\text { Events } \\
\text { Placebo I Nintedanib }\end{array}$ & HR (95\% Cl) \\
\hline \multicolumn{4}{|l|}{ Overall survival } \\
\hline Overall LUME-Colon 1 population $(\mathrm{N}=735)$ & $367 \mid 368$ & $286 \mid 306$ & $1.04(0.88-1.22)$ \\
\hline \multicolumn{4}{|l|}{ Tumor tissue } \\
\hline CMS cohort $(n=231)$ & $114 \mid 117$ & $86 \mid 90$ & $0.87(0.65-1.17)$ \\
\hline Mutations I CNV cohort $(n=157)$ & $84 \mid 74$ & $61 \mid 60$ & $0.97(0.67-1.39)$ \\
\hline cfDNA ( $\mathrm{n}=192)$ & $88 \mid 104$ & $66 \mid 85$ & $0.96(0.69-1.33)$ \\
\hline Protein biomarker $(\mathrm{n}=735)$ & $367 \mid 368$ & $286 \mid 306$ & $1.04(0.88-1.22)$ \\
\hline \multicolumn{4}{|l|}{ Progression-free survival } \\
\hline Overall LUME-Colon 1 population $(\mathrm{N}=735)$ & $367 \mid 368$ & $325 \mid 334$ & $0.66(0.57-0.78)$ \\
\hline \multicolumn{4}{|l|}{ Tumor tissue } \\
\hline CMS cohort $(n=231)$ & $114 \mid 117$ & $98 \mid 110$ & $0.60(0.45-0.79)$ \\
\hline Mutations I CNV cohort $(n=157)$ & $83 \mid 74$ & 72 | 68 & $0.60(0.43-0.85)$ \\
\hline cfDNA ( $\mathrm{n}=192)$ & $88 \mid 104$ & $74 \mid 102$ & $0.46(0.33-0.65)$ \\
\hline Protein biomarker $(\mathrm{n}=735)$ & $367 \mid 368$ & $325 \mid 334$ & $0.66(0.57-0.78)$ \\
\hline
\end{tabular}

Abbreviations: cfDNA = Cell-free DNA; $\mathrm{Cl}=$ confidence interval; $\mathrm{CMS}=$ consensus molecular subtype; CNV = copy number variation; $\mathrm{HR}=$ hazard ratio.

\section{Supplemental Table 5 Distribution and Clinical Characteristics of CMS Subgroups (Pooled Nintedanib and Placebo Arms)}

\begin{tabular}{|c|c|c|c|c|c|c|c|c|c|}
\hline \multirow{3}{*}{$\begin{array}{l}\text { CMS Category } \\
\text { CMS1 }(N=4)\end{array}$} & \multirow{2}{*}{\multicolumn{2}{|c|}{ Sample Site, n (\%) }} & \multirow{2}{*}{\multicolumn{2}{|c|}{ Sidedness, n (\%) }} & \multicolumn{5}{|c|}{ Incidence of Mutations, $\mathbf{n}$ (\%) } \\
\hline & & & & & \multirow[b]{2}{*}{ Samples } & \multirow{2}{*}{$\begin{array}{c}\text { KRAS } \\
3\end{array}$} & \multirow[t]{2}{*}{ BRAF } & \multirow[t]{2}{*}{ APC } & \multirow[t]{2}{*}{ TP53 } \\
\hline & Primary & $2(50.0)$ & Right & $0(0.0)$ & & & & & \\
\hline & Metastasis & $2(50.0)$ & Left & $0(0.0)$ & Mutations & $0(0.0)$ & $0(0.0)$ & $3(100.0)$ & $1(33.3)$ \\
\hline & NA & $0(0.0)$ & NA & $4(100.0)$ & & & & & \\
\hline \multirow[t]{3}{*}{ CMS2 $(\mathrm{N}=64)$} & Primary & $50(78.1)$ & Right & 8 (12.5) & Samples & 39 & & & \\
\hline & Metastasis & $11(17.2)$ & Left & $31(48.4)$ & Mutations & $14(35.9)$ & $1(2.6)$ & $33(84.6)$ & $15(38.5)$ \\
\hline & NA & $3(4.7)$ & NA & $25(39.1)$ & & & & & \\
\hline \multirow[t]{3}{*}{ CMS3 $(N=2)$} & Primary & $2(100.0)$ & Right & $0(0)$ & Samples & 1 & & & \\
\hline & Metastasis & $0(0.0)$ & Left & $1(50.0)$ & Mutations & $0(0.0)$ & $0(0.0)$ & $1(100.0)$ & $0(0.0)$ \\
\hline & NA & $0(0.0)$ & NA & $1(50.0)$ & & & & & \\
\hline \multirow[t]{3}{*}{ CMS4 (N = 119) } & Primary & $96(80.7)$ & Right & $11(9.2)$ & Samples & 75 & & & \\
\hline & Metastasis & $17(14.3)$ & Left & $54(45.4)$ & Mutations & $33(44.0)$ & $0(0.0)$ & $47(62.7)$ & $39(52.0)$ \\
\hline & NA & $6(5.0)$ & NA & $54(45.4)$ & & & & & \\
\hline \multirow[t]{3}{*}{ Unclassified/mixed phenotype ( $\mathrm{N}=42)$} & Primary & $32(76.2)$ & Right & $4(9.5)$ & Samples & 25 & & & \\
\hline & Metastasis & $7(16.7)$ & Left & $18(42.9)$ & Mutations & $9(36.0)$ & $0(0.0)$ & $18(72.0)$ & $12(48.0)$ \\
\hline & NA & $3(7.1)$ & NA & $20(47.6)$ & & & & & \\
\hline
\end{tabular}

Abbreviations: $\mathrm{CMS}=$ Consensus molecular subtype; $\mathrm{NA}=$ not available. 


\section{Biomarker Analysis: Nintedanib in LUME-Colon 1}

Supplemental Table 6 Predictive Analysis of GMS Subgroups

\begin{tabular}{|c|c|c|c|c|}
\hline & \multicolumn{2}{|c|}{ CMS4 } & \multicolumn{2}{|c|}{ Other ${ }^{\mathrm{a}}$ (Non-CMS4) } \\
\hline & Placebo & Nintedanib & Placebo & Nintedanib \\
\hline \multicolumn{5}{|l|}{ Overall survival } \\
\hline Number of patients, N (\%) & $59(100.0)$ & $60(100.0)$ & $55(100.0)$ & $57(100.0)$ \\
\hline OS events, $n(\%)$ & $41(69.5)$ & $48(80.0)$ & $45(81.8)$ & $42(73.7)$ \\
\hline Median time to event, months $(95 \% \mathrm{Cl})^{\mathrm{b}}$ & $6.4(4.5-8.4)$ & $7.5(5.3-9.5)$ & $6.6(4.4-8.0)$ & $6.9(6.2-8.9)$ \\
\hline Nintedanib vs. placebo, HR $(95 \% \mathrm{Cl})^{\mathrm{C}}$ & \multicolumn{2}{|l|}{$0.92(0.60-1.41)$} & \multicolumn{2}{|c|}{$0.81(0.53-1.25)$} \\
\hline Interaction $P$-value ${ }^{\mathrm{d}}$ & \multicolumn{4}{|c|}{.6634} \\
\hline \multicolumn{5}{|l|}{ Progression-free survival (central assessment) } \\
\hline Number of patients, $\mathrm{N}(\%)$ & $59(100.0)$ & $60(100.0)$ & $55(100.0)$ & $57(100.0)$ \\
\hline PFS events, n (\%) & $50(84.7)$ & $55(91.7)$ & $48(87.3)$ & $55(96.5)$ \\
\hline Median time to event, months $(95 \% \mathrm{Cl})^{\mathrm{b}}$ & $1.3(1.3-1.4)$ & $2.2(1.4-2.7)$ & $1.4(1.3-1.4)$ & $2.6(1.4-2.7)$ \\
\hline Nintedanib vs. placebo, HR $\left(95 \%\right.$ Cl) ${ }^{C}$ & \multicolumn{2}{|l|}{$0.60(0.40-0.91)$} & \multicolumn{2}{|c|}{$0.56(0.37-0.85)$} \\
\hline Interaction $P$-value ${ }^{\mathrm{d}}$ & \multicolumn{4}{|c|}{.8278} \\
\hline
\end{tabular}

Abbreviations: $\mathrm{Cl}=$ Confidence interval; $\mathrm{CMS}=$ consensus molecular subtype; $\mathrm{HR}=$ hazard ratio; $\mathrm{OS}=$ overall survival; PFS = progression-free survival.

${ }^{\mathrm{a}}$ Other includes all evaluable patients not classified as CMS4.

bestimated from an unadjusted Kaplan-Meier curve for each treatment arm.

${ }^{\mathrm{c}} \mathrm{HR}<1$ favors nintedanib; model adjusted for treatment, regorafenib pre-treatment, time from onset of metastatic disease until randomization, and region.

${ }^{\mathrm{d}}$ Model adjusted for the 3 stratification factors: treatment, subgroup, and treatment by subgroup interaction. 